\title{
TOTAL SOLAR ECLIPSE
}

OF

\author{
JUNE 8, 1918.
}

B 353 
. REPORT

OF THE

\section{U. S. NAVAL OBSERVATORY ECLIPSE EXPEDITION}

TO OBSERVE THE

\section{TOTAL SOLAR ECLIPSE OF JUNE 8, 1918,}

AT

BAKER, OREGON. 


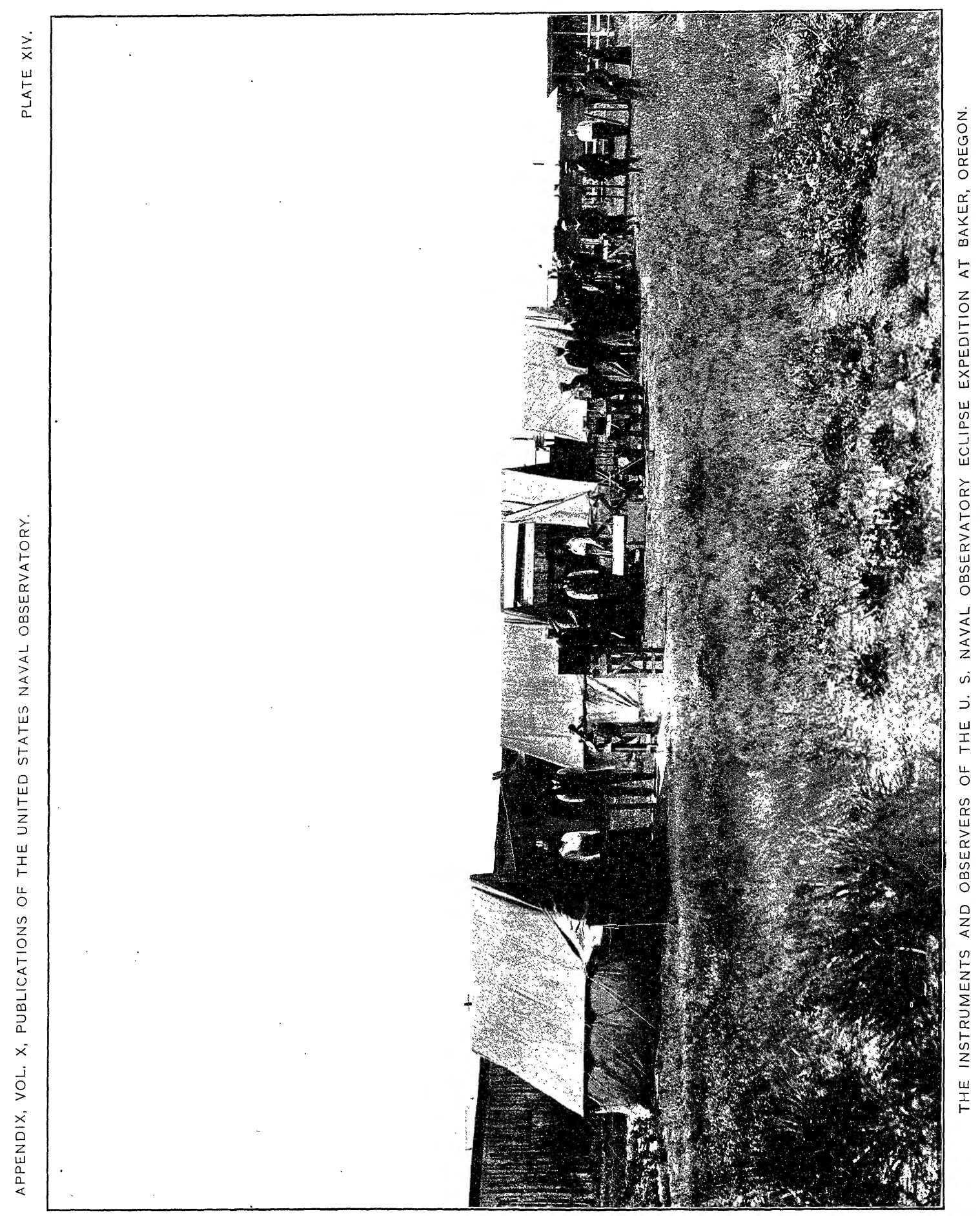




\section{GENERAL REPORT \\ OF THE \\ ECLIPSE EXPEDITION IN 1918.}

Astronomer J. C. HАMмоND, in charge.

The observation of the total solar eclipse of June 8, 1918, by the Naval Observatory, was made possible by a special appropriation of $\$ 3,500$ by Congress. The path of the eclipse crossed the United States in a southeasterly direction from the State of Washington to Florida. Totality occurred at about 2:55 p. m., Pacific standard time, on the western coast of Washington, and at about 6:45 p. m., Eastern standard time, on the eastern coast of Florida. After considering various sites, it was decided to locate at Baker, Oregon, situated very near the center of the path. The weather conditions were likely to be good at Baker in June; also the altitude of the Sun at the time of eclipse was greater, and the duration of totality longer, than at points farther east.

All the preparations were maae during the winter of 1917-18 and the freight car containing the apparatus left Washington on April 11, 1918. The advance party, consisting of Astronomer J. C. Hammond, Assistants C. C. Wylie and W. A. ConRad, of the Naval Observatory, and Dr. S. A. Mitchell, Director of the Leander McCoRMick Observatory of the University of Virginia, arrived at Baker on April 29. They were joined on May 1 by Prof. L. G. Hoxton, of the University of Virginia. These five members of the expedition set up and adjusted the various instruments. They were aided in this work by a party of six men sent from the receiving ship at Puget Sound, Washington. This party consisted of PATrick Welsh, chief quartermaster, N. R. F., W. L. Veale, C. M. 1c., U. S. N., G. J. Pehling, M. M. 2c., N. R. F., and H. H. Herrick, Carl Krummel, and F. Olesen, seamen, U. S. N. These men reported on May 5 and remained until the instruments were all repacked for shipment back to Washington after the eclipse. Not only were they of great aid in erecting the various apparatus, but they also assisted with the different instruments in observing the eclipse.

Other members of the eclipse party arrived as follows: Assistant Astronomer G. H. Peters, of the Naval Observatory, on May 24; Dr. Paul W. Merrill, of the Bureau of Standards, Mr. Edward D. Adams and Kempton Adams, of New York, and Mr. Howard Russell Butler, of Princeton, N. J., on May 31; finally Miss Harriet Bigelow and Miss Mary M. Hopkins, professors of astronomy at Smith College, on June 2.

Baker is a thriving city of 9,000 inhabitants, on the main line of the Union Pacific system to Portland. It is situated in the Powder River Valley between two mountain ranges, the Blue or Elkhorn Range on the west, and the Powder River or Eagle Mountains on the northeast. Its altitude is about 3,400 feet, and the region 
is devoted chiefly to mining, lumbering, stock and fruit raising. Very comfortable quarters for the party were secured at the Hotel Antlers. Through the kindness of Mr. C. L. Palmer, the mayor of Baker, and of Mr. S. O. Correll, an ideal site for the station was obtained at the fairgrounds, which were located about a mile from the center of the city. These grounds were level, afforded ample room for the erection of the various instruments, and contained buildings in which could be stored much of the valuable apparatus when not in use.

Arrangements had been made with the Western Union Telegraph Co. for the use of their lines for an exchange of longitude signals between the eclipse station and the Naval Observatory, and two pairs of wires were run to the station by the Western Union Co. These wires were also used for receiving the daily noon signals from Mare Island. A wireless receiving set, loaned by the Washington Navy Yard, was installed, but no wireless signals were received. This was of little consequence, as the longitude exchange by the Western Union left nothing to be desired.

Preliminary determinations of the longitude and latitude of the station from sextant observations of the Sun by Doctor Mitchell and Mr. Wylie gave as results:

Latitude $=+44^{\circ} 47^{\prime} .9$.

Longitude $=7^{\mathrm{h}} 51^{\mathrm{m}} 24^{\mathrm{s}} .3$ west of Greenwich.

On the night of May 1, a meridian was laid out by observations of Polaris, made with a surveyor's transit borrowed from the local county surveyor, as the eclipse instruments had not yet arrived.

On May 6 the car containing the apparatus arrived safely, and the work of erecting the various instruments went forward rapidly. The weather during May was ideal for this work. The mornings and nights were usually clear, but in the afternoon clouds frequently formed over the Blue Mountains on the west and drifted over the sky, which would often be overcast at 4 o'clock, the time of day when the eclipse was to take place. Windstorms, which filled the air with dust, were also prevalent in the afternoons. No rain fell during the six or seven weeks that the party was there.

The final longitude and latitude of the eclipse station were determined in May. The instrument for determining the local time at Baker was a FAUTH transit of 3 inches aperture, which was equipped with a latitude level and was used also for the determination of the latitude. The observations for time were made by Mr. HAMmond and for latitude by Mr. WYLIE. The observations for time at the Naval Observatory were made by Assistant MATT FREDERICKson with the 6-inch transit circle. A direct exchange of signals between the eclipse station and the Naval Observatory was made over the lines of the Western Union Telegraph Co. on four nights. The resulting values of the longitude and latitude are given in the report of Mr. Wrlie.

EQUIPMENT AT BAKER, OREGON.

The 5-inch equatorial.-A CLARK equatorial of 5 inches aperture was used to observe the times of contacts. This instrument was also used on many nights in May to show objects of interest in the heavens to the people of Baker and vicinity, 
who took advantage of this opportunity in great numbers, as many as 200 persons visiting the station on some nights.

The 65-foot camera.-This camera, which was used to photograph the inner corona, was horizontal and was directed toward the sunrise point. Light from the corona was reflected from a silvered glass mirror, 10 inches in diameter, attached to a coelostat controlled by clockwork. The lens, which was 7.5 inches in aperture and 65 feet in focal length, is described in detail in the report of the total solar eclipse of August 30,1905, which is contained in the present volume. The coelostat was made by Brashear and is described in the Publications of the U. S. Naval Observatory, Second Series, Volume IV, Appendix I.

The camera tube and building were constructed entirely of wood and were covered on the inside with building paper to prevent light leaking through. The piers for supporting the plate-holder and lens were also made of wood and set in the ground in cement. These piers were entirely independent of the building and tube. An awning of wood was constructed over the tube to act as a rain and sunshade.

The shutter for the camera was the same as previously used by Mr. G. H. Peters on three former eclipse expeditions. It was arranged and operated in the same manner as described by him in the report of the 1905 eclipse station at Porta Coeli, Spain.

Considerable irregularities were found to exist in the worm wheel of the Brashear coelostat, and the most nearly perfect part was selected for use during the eclipse.

The photographic plates were double coated, special coronal, 17 by 20 inches, made by the Eastman Kodak Co. The diameter of the Sun's image on the plate is about 7.25 inches.

Five exposures were made of $5,15,35,15$, and 5 seconds' duration, respectively. This instrument was in charge of Mr. ConRAD, who was assisted during the eclipse by W. L. Veale.

The 105-inch camera.- This camera, which was used to photograph the middle portions of the corona, had a visual lens 6 inches in aperture and 105 inches in focal length. It was used with a color screen and 8 by 10 inch CRAmer isochromatic plates. It was also horizontal, and light from the corona was reflected to it from a silvered glass mirror 7 inches in diameter attached to a GAERTNER coelostat. Three exposures were made of 15, 50, and 25 seconds' duration. The camera was in charge of Mr. Peters, who was assisted during the eclipse by CarL Krummel.

The 33-inch camera.-This camera had a DALLMEYER portrait lens of 6 inches aperture and 33 inches focal length. A GaERTNER coelostat, controlled by clockwork, carried a 10-inch silvered glass mirror for this camera at its upper end and the mirror for the 105-inch camera at its lower end. It was also horizontal, and double-coated special coronal plates 5 by 7 inches were used. Two exposures were made of 65 and 40 seconds' duration to secure the outer extensions of the corona. This camera was in charge of Mr. WyLIE, who was assisted during the eclipse by H. H. HerRick.

101141 -26-PT II- -61 
The spectrographs. - The spectroscopic work was under the supervision of Doctor Mitchell, who had been a member of the Naval Observatory Eclipse Expeditions of 1900,1901 , and 1905. Three spectrographs were used, each equipped with a concave grating and used without slit. Each was mounted horizontally, and light was fed to the grating by a silvered glass mirror 7 inches in diameter attached to a coelostat. A detailed description of these spectrographs is contained in the report of Doctor Mitchell. Plate XIV shows the various instruments and observers.

ECLIPSE DAY.

On the morning of June 8 the sky was entirely overcast. The limb of the Sun could just be discerned through the equatorial. About noon it began to clear somewhat. At the beginning of the eclipse there was a thin cloud over the Sun, but the first contact was easily observed. As totality approached there were clear patches of sky near the Sun, and the clouds were moving in such a way that it seemed certain that the solar disk would be in a clear space during totality. This expectation was not realized, however, as a light cloud covered the Sun during totality. Five minutes later the Sun was in a clear space.

The seeing was very steady and the photographs, while they do not show the outer and fainter extensions of the corona, give fine representations of the inner corona and prominences. A full discussion of the negatives is contained in the report of Mr. Peters.

Contacts.-The first three contacts were observed by Mr. Hammond, who also gave the signal for starting after the second contact. The first contact was estimated to be late by 3 or 4 seconds, but the second and third contacts were well observed. The observation of the last contact was impossible as it was again cloudy. The observed and computed times of the first three contacts are as follows:

Greenwich Mean Times of Contacts.

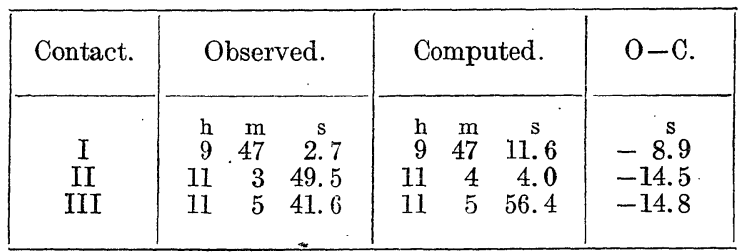

The computed times are derived from data in the American Ephemeris. The correction to the computed times for Baker, as given by Assistant ArTHur NewTon, of the Nautical Almanac Office, in A. J. 733, is - 12.5 seconds. The computed duration of totality was 112.4 seconds and the observed duration 112.1 seconds.

Professor Howard, of the physics department of the Baker High School, and Professor Whitman, of the same department in the Pendleton High School, volunteered to observe the shadow bands. None were seen, howèver. Mr. Kempton Adams photographed the eclipse with two small cameras, pointed directly at the Sun. Patrick Welsh counted the seconds during totality to guide the various observers in making the exposures. 
A novel feature of this eclipse was the painting of the corona by Mr. Howard Russell Butler. The picture of the eclipse, painted by this noted artist from shorthand sketches made during the short period of totality, is one of great interest and beauty. (See Frontispiece.)

An interesting occurrence was the appearance of the brilliant new star in Aquila. This star was seen by Mr. Peters and Mr. Conrad on the night of June 8 while they were developing the eclipse negatives, and the discovery was communicated by telegram to various observatories in the country. It had, however, been discovered earlier on the same night by other astronomers in the East.

Special mention should be made of the interest taken in the expedition and the assistance rendered it by the people of Baker. In particular, thanks are due to Mr. C. L. Palmer, mayor of Baker, and to Mr. W. Meacham, secretary of the Commercial Club, for the many courtesies extended by them, to the Fairground Association for the use of their grounds and buildings, and to Mr. L. A. Tibbals, who gave the use of a small building in which were kept the chronometers, chronograph, wireless set, and other valuable apparatus. It is desired also to express appreciation to Representative C. N. McARTHuR, of Portland, for the keen interest which he took in the success of the expedition.

\section{DETERMINATION OF THE LONGITUDE AND LATITUDE AT BAKER, OREGON.}

By Assistant Charles Clayton Wylie.

The following instruments were used to determine the longitude and latitude of the station at Baker, Oregon, chosen by the U. S. Naval Observatory party, for observation of the eclipse of June 8, 1918:

FAUTH transit and zenith telescope 1944; sextant BrANDIs 3257; sidereal breakcircuit chronometer NeGUs 1518; sidereal break-circuit chronometer NEGUs 1902; mean time break-circuit chronometer NARDin 589; mean time watch, Hamilton U. S. N. 1758.

\section{PRELIMINARY DETERMINATION.}

The sextant and watch, as well as the chronometers, were carried as hand baggage, and the former were used before the arrival of the transit in a preliminary determination of the position of the station. During this work the watch was compared daily with the time signals from Mare Island as received at the local Western Union office. Observations for longitude were made by equal altitudes, using the method of the Magnetic Division of the U. S. Coast and Geodetic Survey. In the forenoon five readings were taken on one limb of the Sun followed immediately by five on the other limb at the same settings. Observations were taken at the same readings in the reverse order in the afternoon. The watch was running on Greenwich mean time, and the reduction of the observations gave directly the Greenwich mean time of local mean noon, or the longitude. Four equal altitude sets were observed by Dr. S. A. Mitchell, Director of the Leander McCormick Observatory, and three by Mr. C. C. WrLIE, of the U. S. Naval Observatory, giving

$$
\text { Longitude }=7^{\mathrm{h}} 51^{\mathrm{m}} 24^{\mathrm{s}} .3 \text { west of Greenwich. }
$$


The preliminary latitude was obtained by circummeridian double altitudes of the Sun. Two sets were obtained by each observer, giving

$$
\text { Latitude }=+44^{\circ} 47^{\prime} 54^{\prime \prime} \text {. }
$$

FINAL DETERMINATION.

The FAUTH transit was used to determine the adopted position. This instrument is of 3 inches aperture and 35 inches focal length. Mr. H. C. Cleve, the U. S. Naval Observatory instrument maker, fitted it with an accurate level for latitude work. The micrometer equivalent was determined at the eclipse station both directly by observations of circumpolar stars and indirectly by determining the thread intervals from the observations for time. The value of one division of the latitude level was determined at the station by mounting an engineer's transit as a collimator. With the level bubble in different positions readings were taken on the reticule of the engineer's transit with the FAUTH micrometer. This gave the value of a level division in terms of the micrometer equivalent. The striding level was tested at Washington in a similar manner, as follows, both before and after the work at Baker: The level, in the rectangular box in which it was used on the FAUTH instrument, was laid on the 6-inch transit circle when that instrument was horizontal and pointing at one of the collimators. With the bubble of the level in different positions, readings were taken on the collimator threads with the micrometer of the transit circle. This gave the value of a division of the striding level in terms of the zenith distance micrometer screw of the transit circle.

Chronometer NEgus 1518 was used as standard. It was compared regularly by chronograph with NeGUs 1902. It was compared by coincidence of beats with NARDIN 589, as the break-circuit of that chronometer was not in good order.

Longitude.-The observations for clock correction at the time of the exchange of signals were made at Baker by Mr. J. C. HAMmond, with the FAUTH transit and at Washington by Mr. MatT Frederickson with the 6-inch transit circle. At Baker zenith stars were selected in groups of five or six, such that the sum of the $A$ factors in a group would be less in absolute, value than 0.1 , thereby minimizing the effect of an error in the adopted azimuth. As additional precaution, an azimuth star with $A$ factor greater than 1.8 was observed with each group. On three of the four nights complete groups were observed at Baker both before and after the exchange of signals, the instrument being reversed between groups, making a total of about twenty zenith and three or four azimuth stars. At Washington the program was to determine the instrumental constants and observe six zenith stars, both before and after the exchange of signals. On one night clouds prevented the observations at Baker after the exchange, and on another clouds prevented the observations at Washington after the exchange. All the observations at Washington were made with the instrument Clamp West.

The Western Union Telegraph Co. had kindly installed a loop from their main line, distant a few hundred yards, so that the daily time signals from Mare Island could be accurately compared by chronograph with the chronometers. On four nights a through line was provided from the U. S. Naval Observatory, Washington, 
D. C., to the eclipse station. The line was cleared for 20 minutes at about $20^{\mathrm{h}}$ Greenwich mean time, or according to the "summer time" then in use, 4 a. $\mathrm{m}$. Eastern time, or 1 a.m. Pacific time. Mr. C. B. WATts, at Washington, and Mr. C. C. WyLIE, at Baker, made the exchange of telegraphic signals for longitude. Before the party left Washington "longitude boards" were designed and constructed, one for use at Washington, the other for use at Baker. They were so designed that the clock or chronometer beats were put on the main line from the secondary of the sending chronograph as breaks. A key was designed for each board to send arbitrary break-circuit signals in a similar manner.

At the time of an exchange the beats of the standard clock at Washington were put on the main line and recorded on the chronograph at Baker along with the beats of the eclipse party chronometer. Then similarly, the beats of the chronometer were put on the main line, and recorded in Washington along with those of the standard clock. Signals were also exchanged by arbitrary breaks. The operator at one station makes breaks in the main-line circuit which are recorded on the chronographs at both stations; then the operator at the other station does the same. This is a better method of determining the longitude than exchange of clock beats only. The entire series of beats of an exchange falling at about the same hundredth of a second are little better than. a single good signal. All are subject to the same personality in estimating the hundredth. Due to lag of line the clock beats are generally not in coincidence at the two stations simultaneously, and when in coincidence at either, the method fails. The arbitrary signals fall in different parts of the second. Personality in reading the record is thereby eliminated and accuracy greatly increased. In spite of over 3,000 miles of line, the records were almost perfect and a rapid comparison was sufficient to identify the breaks and reject all not common to both.

\begin{tabular}{|c|c|c|c|c|c|c|}
\hline \multirow{2}{*}{ Date. } & \multicolumn{4}{|c|}{ Longitude from Washington. } & \multicolumn{2}{|c|}{$\begin{array}{c}\text { Double Transmission } \\
\text { Time. }\end{array}$} \\
\hline & \multicolumn{2}{|c|}{ Arbitrary Signals. } & \multicolumn{2}{|c|}{ Clock Beats. } & $\begin{array}{l}\text { Arbitrary } \\
\text { Signals. }\end{array}$ & $\begin{array}{l}\text { Clock } \\
\text { Beats. }\end{array}$ \\
\hline $\begin{array}{r}1918 \\
\text { May } 16 \\
17 \\
22 \\
23\end{array}$ & $\begin{array}{lr}\mathrm{h} & \mathrm{m} \\
2 & 43\end{array}$ & $\begin{array}{c}\mathrm{s} \\
7.21 \\
7.23 \\
7.28 \\
7.22\end{array}$ & $\begin{array}{lr}\mathrm{h} & \mathrm{m} \\
2 & 43\end{array}$ & $\begin{array}{l}\mathrm{s} \\
7.21 \\
7.22 \\
7.27 \\
7.23\end{array}$ & $\begin{array}{c}\mathrm{s} \\
0.39 \\
0.38 \\
0.45 \\
0.38\end{array}$ & $\begin{array}{c}\mathrm{s} \\
0.39 \\
0.36 \\
0.48 \\
0.39\end{array}$ \\
\hline Mean & 243 & $7.23 \pm 0^{s} .01$ & 243 & 7.23 & & \\
\hline
\end{tabular}

The probable error was determined by intercomparing the results on the different nights among themselves. The mean obtained, giving half weight to the two nights on which observations were weak, is the same as the above result. On May 21 a storm put many of the Western Union lines out of order in the Middle West. Perhaps this necessitated a different routing of the line on May 22, as the transmission time is longer than on the other nights. Assuming the longitude of the U. S. Naval Observatory given in the American Ephemeris, we have for the adopted longitude 
It should be stated that the relative personal equation of the observers was not allowed for in obtaining this result. The 6-inch transit circle is equipped with a self-registering micrometer in right ascension. The personal equation of $\mathrm{Mr}$. FREDERICKSON is therefore negligible. Before going to Baker, observations were made at Washington with the $\mathrm{F}_{\mathrm{AUTH}}$ transit to determine relative personal equation. Two nights by Mr. HAMmond gave $+0^{\mathrm{s}} .06$ and $+0^{\mathrm{s}} .12$ and the mean of four nights by Mr. WyLIE gave $+0^{\mathrm{s}} .15$ to be applied to chronometer corrections. The plan, in preparing for the expedition, was that Mr. WYLIE should make the observations for the longitude work, but a minor accident partially incapacitating him just as the work was being started, Mr. HAMmond made the longitude observations.

Latitude.-The observations for latitude were made by Mr. WruIE on four nights, using the FAUTH transit as a zenith telescope. Twenty-four different pairs of stars, selected from the General Catalogue of Boss, were observed, some on more than one night.

\begin{tabular}{|r|rr|r|r|}
\hline Date. & \multicolumn{2}{|c|}{ Latitude. } & $\begin{array}{r}\text { Number } \\
\text { of Pairs. }\end{array}$ \\
\hline 1918 & & & & \\
\hline May 27 & +44 & 47 & 25.94 & \\
28 & & & 25.42 & 5 \\
29 & & & 26.00 & 8 \\
31 & & & 25.79 & 11 \\
& & & \\
\hline
\end{tabular}

The adopted latitude was obtained by taking the weighted mean of the results from the different pairs of stars. The result of one good observation of a pair was weighted two; two good observations, three; and three good observations, four. This gave for the latitude

$$
+44^{\circ} 47^{\prime} 25^{\prime \prime} .83 \pm 0^{\prime \prime} .14 \text {. }
$$

The adopted position of the eclipse station at Baker, determined with the FAUTH transit and zenith telescope, is therefore

$$
\begin{aligned}
& \text { Longitude }=7^{\mathrm{h}} 51^{\mathrm{m}} 23^{\mathrm{s}} .01 \text { west of Greenwich; } \\
& \text { Latitude }=+44^{\circ} 47^{\prime} 25^{\prime \prime} .83 \pm 0^{\prime \prime} .14 \text {. } \\
& \text { COMPARISON AND GENERAL REMARKS. }
\end{aligned}
$$

As the sextant and adopted results do not agree as well as might be anticipated,

\begin{tabular}{|c|c|c|c|c|}
\hline Date. & Observer. & \multicolumn{2}{|c|}{ Longitude. } & Sextant- \\
\hline $\begin{array}{l}1918 \text { May } 1 \\
3 \\
3\end{array}$ & $\begin{array}{l}\text { WYLIE . . } \\
\text { WYLIE . } \\
\text { WYLIE . }\end{array}$ & $\begin{array}{ll}\mathrm{h} & \mathrm{m} \\
7 & 51\end{array}$ & $\begin{array}{c}\mathrm{s} \\
23.85 \\
24.18 \\
23.72\end{array}$ & $\begin{array}{c}\mathrm{s} \\
+0.84 \\
+1.17 \\
+0.71\end{array}$ \\
\hline $\begin{array}{l}\text { Mean (3 sets) } \\
\text { Mean (4 sets) }\end{array}$ & $\begin{array}{l}\text { Wylie . . . . . } \\
\text { Mitchell. }\end{array}$ & \multicolumn{2}{|r|}{$\begin{array}{l}23.92 \\
24.66\end{array}$} & $\begin{array}{l}+0.91 \\
+1.65\end{array}$ \\
\hline
\end{tabular}
let us examine some of the sextant work in detail. The equal altitude sets for longitude by Mr. WYLIE, and the mean of the same, and the mean of Dr. Mitchell's sets are 
This shows a large systematic deviation. Instrumental errors theoretically have little effect with this method. The rate of the watch was highly satisfactory throughout the stay in Baker and later tests indicated it could be compared with the Mare Island time signals as transmitted by the Western Union Telegraph Co. with a probable error under a tenth of a second. Also, the eccentricity of the second hand dial was found to be negligible. Possible sources of systematic error are the signal itself and the method of its transmission. Throughout the periods when observations for time were obtained on the FAUTH transit the correction and rate to the standard sidereal chronometer were obtained from the observations. The daily time signals were compared by chronograph with this chronometer and, assuming the adopted longitude, corrections to the time signals as received were computed. They varied from $-0^{\mathrm{s}} .48$ on May 22 to $+0^{\mathrm{s}} .63$ on June 7 . The correction usually changed slowly from day to day, corrections on successive days being about the same. As the sextant longitude sets were taken May 1 to May 3, inclusive, it is possible the signal had a large systematic error on those three days. The difference between the two observers (MITCHELL-WYLIE $)=+0^{\mathrm{s}} .74$, suggest that with the low magnifying power of the sextant there is a large personal equation.

The sextant results for latitude also show a systematic deviation. This was noted when a preliminary reduction of the latitude work with the FAUTH instrument was made. The sextant was readjusted and a set of circummeridian double altitudes observed on June 10, 1918. Let us examine in detail this set, which agrees substantially with the earlier work. Readings for index correction made before and after observing gave a probable error $\pm 2^{\prime \prime} .5$. Ten circummeridian double altitudes, five on each limb of the Sun, gave the following results for latitude:

\begin{tabular}{|c|c|}
\hline Lower Limb. & Upper Limb. \\
\hline $\begin{array}{rrr}\circ & \prime & \prime \prime \\
44 & 47 & 59 \\
47 & 39 \\
48 & 14 \\
48 & 5 \\
47 & 50\end{array}$ & $\begin{array}{rrr}\circ & , \quad \text { " } \\
44 & 47 & 31 \\
47 & 40 \\
47 & 29 \\
47 & 33 \\
47 & 24\end{array}$ \\
\hline Mean (10 readings & $7^{\circ} 47^{\prime} 45^{\prime \prime} \pm 3^{\prime \prime} .6$ \\
\hline
\end{tabular}

Combining this probable error of the double altitude measures with that of the index correction, we obtain $\pm 4^{\prime \prime} .4$. But the mean latitude is $19^{\prime \prime}$ from the adopted latitude.

All sets for latitude taken with the sextant are:

\begin{tabular}{|c|c|c|c|c|}
\hline Date. & Observer. & Latitude. & Weight. & Remarks. \\
\hline $\begin{array}{rr}1918 \text { May } \\
\text { May } \\
\text { May } \\
\text { June } 10 \\
\text { Weighted mean }\end{array}$ & 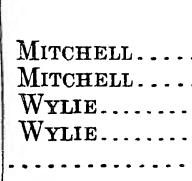 & $\begin{array}{rrr}\circ & \prime & \prime \prime \\
44 & 47 & 56 \\
48 & 7 \\
47 & 55 \\
47 & 45 \\
44 & 47 & 54 \pm 2^{\prime \prime} .9\end{array}$ & $\begin{array}{l}2 \\
1 \\
1 \\
2\end{array}$ & $\begin{array}{l}\text { Good. } \\
\text { Clouds interfered. } \\
\text { Clouds interfered. } \\
\text { Good. }\end{array}$ \\
\hline
\end{tabular}


This mean differs $28^{\prime \prime}$, or nearly ten times the probable error, from the adopted latitude. The agreement of the sets taken by the two observers might point to an error in the sextant. A correction for eccentricity of about $12^{\prime \prime}$, furnished with the sextant, was applied.

The local weather bureau was perhaps a mile from the eclipse station and at about the same elevation. It was in charge of Mr. Maxwell, who kindly furnished special readings of the thermometer and barometer for the computation of the refraction for the sextant latitude work. Comstock's formula (CAMPBELL's Practical Astronomy, p. 35),

$$
r=\frac{983 b}{460+t} \tan z,
$$

in which $b$ is the pressure in inches, $t$ the temperature of the atmosphere in degrees $\mathrm{F}$., and $z$ the apparent zenith distance, was used. The formula was tested by computing the refraction for two of the extreme altitudes by the Pulkowa tables. The results differed by less than $0^{\prime \prime} .1$. The quantities for one of the altitudes tested were

$$
z=29^{\circ} 33^{\prime} .0, t=70^{\circ}, b=26.46 \text { inches. }
$$

It is of interest that the formula holds well for a low barometer.

The eclipse station was located in the fairgrounds about one mile northwest of the central portion of Baker. The concrete pier of the FAUTH instrument is the point for which the longitude and latitude were accurately determined. The owners of the grounds seemed pleased to preserve this to mark the site of the eclipse station. In the top of the pier is embedded a brass tablet engraved

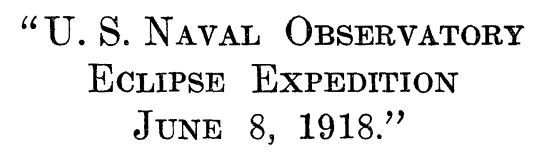

\section{REPORT OF ASSISTANT ASTRONOMER G. H. PETERS.}

Acting under orders from the Navy Department, I left the Naval Observatory for Baker, Oregon, on May 20, 1918, as a member of the party to observe the total solar eclipse, arriving at Baker on May 25. Before the expedition left Washington I had performed considerable preliminary work on the equipment. This involved securing photographic and chemical supplies, silvering the coelostat mirrors, etc. My departure for the station, however, had been delayed as long as possible on account of war work, upon which I was actively engaged at this period. The instrumental installation was nearly completed upon my arrival at Baker. The dark-room arrangements and some minor work on the instruments, including accurate focal settings, was practically all the unfinished work at the station upon my arrival. After the completion of these details frequent drills in the simultaneous operation of all the instruments at the station, in practice for the eclipse program, were carried on daily.

This was the fourth Naval Observatory eclipse expedition of which I had been a member. Previously I had been in charge of and had operated the long-focus cameras at the stations to which I was attached. As this work had necessitated 
$\mathrm{E}$

N

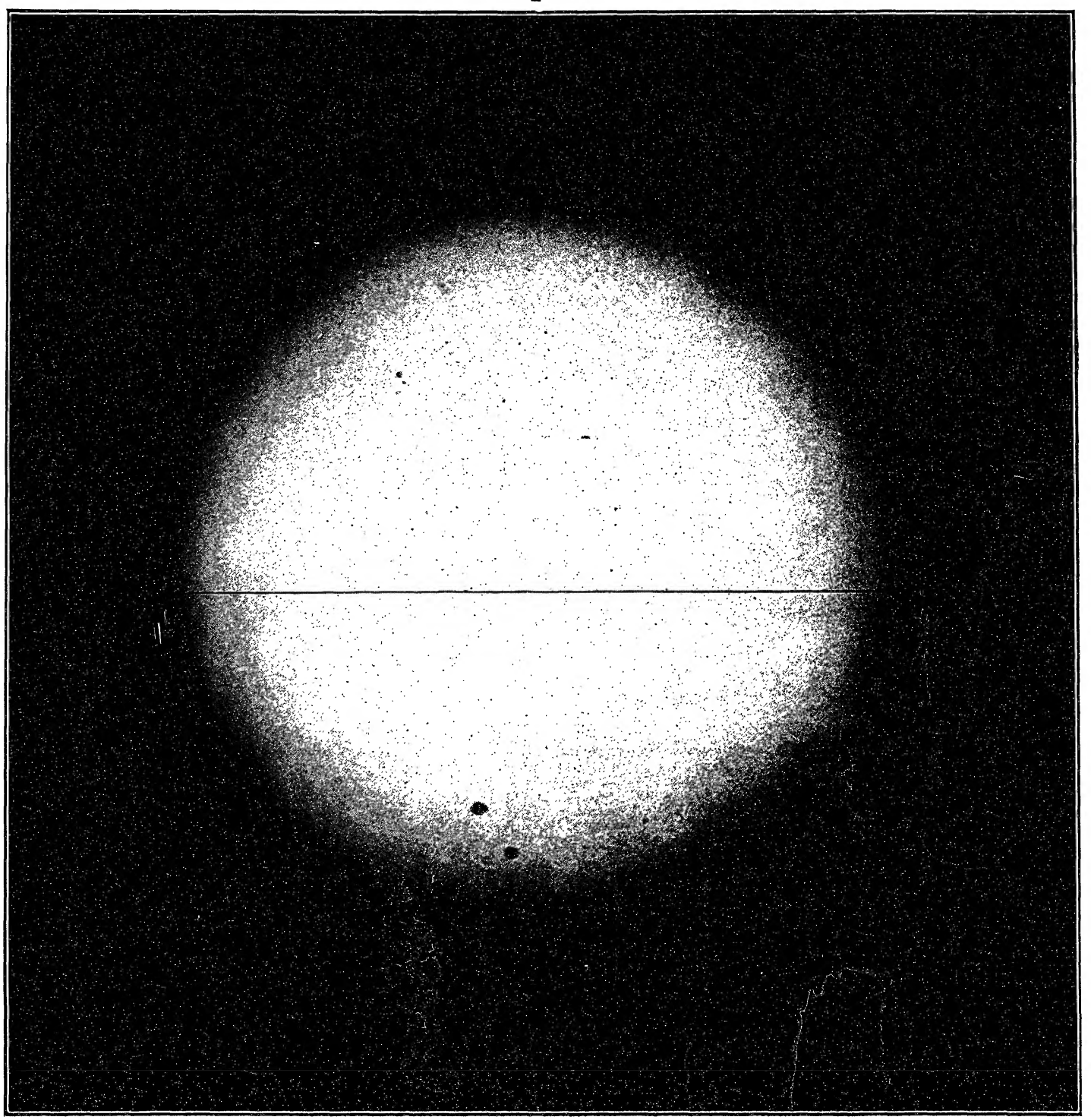

PHOTOGRAPH OF THE SUN SHOWING SUN-SPOTS. TAKEN AT NOON, U. S. NAVAL OBSERVATORY, WASHINGTON, D. C., JUNE 8, 1918, WITH THE 40-FOOT PHOTOHELIOGRAPH. 
my remaining in the dark room of the large instrument at totality, I had never obtained a direct view of an event of this kind. I had intended to act in the same manner upon this occasion. As Mr. CoNRAD had been very zealous in supervising the erection of the 65 -foot camera at the station before my arrival and was willing to take charge of the same for the eclipse observations, I considered it only fair that he should be assigned to this instrument.

The 6-inch visual lens, with color screen, which had always given fine results, was placed in my charge. This 6-inch lens had a focal length of about 105 inches, the Moon's image being nearly an inch in diameter. The function of this instrument was to obtain photographs of the middle corona with differently timed exposures and to show detail over a considerable range of extension. The plates used were 8 by 10 inches CRAMER isochromatic instantaneous. These had been employed on previous occasions with this apparatus with fine results. The color screen was filled with a dilute solution of picric acid in water for absorbing the blue rays of the secondary spectrum of the visual lens and was located inside the tube of the camera about a foot in front of the plate. In this position small floating particles in the solution would not affect the details of the image. The mounting of the screen also acted as a diaphragm, preventing reflections from the sides of the camera box reaching the sensitive plate.

Owing to the shortness of totality, but three exposures were planned for this visual lens. These were to be of 15,50 , and 25 seconds, respectively, and the program on this basis was carried out successfully.

Although the sky was partly clear at totality, a thin cloud veiled the solar surroundings at that time. This cloudiness reduced the amount of light received from the corona, rendering observations of the coronal extensions impossible. It also reduced the contrast of the coronal features, so that detail was unavoidably lost on these plates at short distances from the Moon's limb.

This was also true, but to a much greater extent, on the photographs made with the camera employing the 6-inch DALLMEYER lens in charge of Mr. C. C. WYLIE. With this instrument of 33-inch focal length it was desired to obtain the extensions of the outer corona. But little detail is shown on these negatives, although a considerable amount of coronal extension was photographed. The inner corona as it extends outward decreases rapidly in brightness. This decrease is more apparent on the photographic plates than it is visually. For that reason to obtain the necessary detail on the negatives large-scale photographs of the inner corona are required. For photographs of the coronal extensions it is necessary that instruments of different relative speeds and focal lengths be used with a variety of exposures.

The thin clouds existing at the time of totality in the region of the Sun were illuminated by diffused light from the solar surroundings. When the amount of light received from any part of the corona has the same intrinsic brightness or is fainter than the clouds through which it is transmitted, the photographic action of the coronal light will produce no relative image or contrast effect.

Although the eclipse plates were thoroughly backed with Winsor \& NEwTon moist lampblack, as usual, the diffuse cloud illumination near the Moon's limb was sufficient to fog the otherwise transparent image of the Moon on most of these 
negatives. Consequently, with the exception of some of the shorter exposures, the Baker negatives are not of the highest value for extent and detail in the corona. The definition of the prominences and correct focusing of the Moon's limb are shown with remarkable sharpness on the exposures made with each instrument.

The brilliancy of the large prominences visible and the purity of color of these solar appendages was a revelation to me when seen directly by the eye. The soft greenish yellow light of the corona tinted with pink on the inner parts and blending into pearly light at the extensions was a beautiful spectacle. The whole, though slightly marred by the thin cloud veil, through which it was seen, will never be forgotten.

The distant snowcapped Elkhorn range of. mountains was just outside the shadow path. They could be seen at totality faintly illuminated by the rays of the partially eclipsed Sun. The degree of darkness of this eclipse at our station was not considerable. The light intensity at mid-totality might be compared to the diffused light of the full Moon veiled behind thin clouds. No difficulty was experienced in manipulating apparatus from want of illumination. The thin clouds in the sky probably added to the diffused light inside the shadow. A certain amount of reflected light from the distant snowcapped mountains would also add to the general illumination at the station. The light still remaining at totality had a weird appearance unlike anything else that appears in nature.

Owing to unusual conditions existing throughout the country, due to the war, some difficulty and delay was experienced in securing supplies for the expedition. Chemicals, photographic plates, and other material were somewhat delayed in delivery. Fortunately, the Naval Observatory had considerable apparatus and supplies on hand, which had been used on former expeditions. It had been determined to use a special and newly devised plate for some of the exposures at this station. The proposed plates were especially sensitive to the green rays in the region of $\lambda 5304$ (Young, General Astronomy, rev. ed., p. 231) the coronium line. This plate had been tentatively produced by Dr. C. E. K. MEES of the research department of the EAstman Kodak Co. Owing to the difficulty and delays in securing these plates, due to war conditions, their use was finally abandoned.

Special coronal double-coated plates were furnished by the EAstman Kodak Co. They were employed exclusively with the 7-inch long focus lens and the 6-inch DALLMEYer lens of 33-inch focal length.

The plates used with the former instrument measured 17 by 20 inches and with the latter 5 by 7 inches.

The 6-inch visual lens, with color screen as previously stated was used with Cramer isochromatic instantaneous plates.

At the Porta Coeli Station, Spain, in 1905, 18 by 22 inch plates were employed for the 65 -foot instrument, which was used there for the first time. It was found that this size of plate was unnecessarily large, for the short exposures given to photographs of the inner corona. Plates 17 by 20 inches, somewhat more convenient to manipulate, were therefore adopted for the Baker station. They proved satisfactory in all respects. 
Developing the plates. - It was realized after the eclipse was over that the presence of the cloud haze necessitated special precautions in developing the negatives. This extra care was necessary to bring out the greatest extent of coronal detail for which each plate on its respective instrument was designed. The so-called tentative process was employed to accomplish these results. Separate mixtures of the developing agents were prepared, varying somewhat in the proportion of ingredients, to give different degrees of contrast in the negatives. These solutions were used according to judgment, individually in each case, as conditions determined and as the development proceeded. The coronal images were first brought out with a weak normal developer until the necessary modifications could be ascertained.

The development of the plates was begun shortly after sunset on the night of June 8, after a hard day's work on the eclipse observations. It was carried on till about 4 o'clock the next morning. Upon the succeeding night the development of all the coronal plates was finished. In this work I was ably assisted by Mr. ConRAD, and thereby considerable time was saved. After fixing, the plates were carried outside the dark room for washing. Here, nearby, plenty of space and running water for this latter process was available. This water supply was very cold and of fine quality. It was obtained from the city water system, originating in a reservoir on a neighboring hill, fed by the melting snows of the surrounding mountains.

Discovery of Nova Aquila, No. 3.-After developing and fixing the eclipse negatives on the night of June 8 , it was necessary, as stated, to carry them outside the dark room, to the washing trays. The night was beautifully clear. Mr. ConRaD and I, viewing the heavens, noticed a bright and unfamiliar star in the constellation Aquila, then near the meridian. Having a set of celestial charts at the station, the object was found to be a Nova, and its right ascension and declination noted. When first seen by us it was about midway in brightness between Altair and Vega. The Nova appeared to have a peculiar pulsating scintillation which was absent in the other two stars mentioned. Gradually the Nova increased in brightness from hour to hour. At dawn it was nearly, but not quite, equal to Vega in brilliancy.

At Baker for several days it was not ascertained that this Nova had been discovered independently by many other persons. A note was published by Mr. Conrad and myself, in Popular Astronomy, Volume XXIV, No. 7, giving the details of our observations on this object.

\section{DISCUSSION OF THE RESULTS FROM THE NEGATIVES.}

The solar prominences on the Baker negatives are to be seen on some of the plates made with each of the three instruments. They are shown to advantage, however, both with respect to size and detail only upon the negatives taken with the 65-foot camera. As the negatives made with this instrument were especially intended for the discussion of the prominences and the inner corona, these, in general, will alone be depended upon for this purpose. As previously stated, no accurate description can be given of the outer corona on account of the prevalence of thin clouds at totality. The middle corona shows but little detail on the negatives made with the 6 -inch visual lens. The extent and outline of all but the fainter extensions are shown. The details of the individual rays and streamers are lost 
through the light-diffusion effects of the thin clouds. The 6-inch DALLMEYER camera shows the corona to about the same distance outward as the 6-inch visual lens but with less detail. Consequently the negatives made with it are of little value in discussing the coronal features. It was intended to observe the outer coronal extensions with the DaLlmeyer lens.

The discussion of the solar surroundings at this eclipse follows the same plan as that employed by the writer in the previous expeditions of the Naval Observatory in 1900, 1901, and 1905. The position angles, as usual, are measured from the north point of the heavens towards the east. The corresponding latitude of the solar limb is then assigned. The location of the position angle of the Sun's pole from data given in the Nautical Almanac is taken as $-12^{\circ} .5$. From the same source the inclination of the north pole is about $0^{\circ} .4$ towards the Earth, a very favorable position for this discussion.

Ordinarily plates taken with these eclipse instruments have been oriented by means of star trails taken at the focus.

The plates in this case made for determining the focus were not returned to the Naval Observatory with the coronal negatives. Consequently other means have been employed for obtaining this orientation.

The plate-holding attachment of the long-focus lens was accurately leveled during the erection of the instrument, and therefore the base of a plate was in the plane of the horizon. The plates used at totality had ground edges. The 65 -foot instrument at the station was directed to the sunrise point of the horizon. From the above data, combined with the latitude and longitude of the station and the hour angle and Sun's declination, the direction of the north point with respect to the plate was furnished by computation by Astronomer HAMmond, chief of the expedition. The general direction of east and west was known from observations of the direction taken by the stars in trailing. The same is also true with respect to the motion of the solar image when the driving clock of the coelostat was stopped. The plates for the negatives to be made during totality were numbered at the top. Therefore the identification of the coordinates of the plates is easily made from this data. East was found to be in the lower left-hand corner of the plate, as seen from the glass side looking towards the lens. West was situated near the upper right-hand corner of the plate.

The computed position of the north point on the negatives makes an angle of $50^{\circ} 10^{\prime}$ with respect to the base of the plate. The direction of this line is upwards and with the apex of the angle at the lower right-hand corner of the plate. A straight line bisecting the center of the Mocn's image has been drawn upon the glass side of the original negatives, representing a circle of declination. Measures for position angles are referred to this line. Negatives 1, 2; 4, and 6 will be used principally in this discussion. Negatives 2 and 4 were made somewhat symmetrically with respect to mid-totality. The short exposures on Negatives 1 and 6 are especially valuable for tracing the prominences as near to their source as possible. The relative motion of the Moon during totality gives an unusual importance to these two negatives for this purpose. 
The inner corona.-The inner corona at this eclipse presents numerous features of unusual interest. These special features will be described under the headings of polar rays and equatorial regions. Among the latter are to be found many coronal arches, for which this eclipse is remarkable. While the positions of the objects described will be given, no approach to refined accuracy in this respect can be expected. It should be noted, as stated by the writer on several previous occasions, that the appearances described are those of a nebulous body of three dimensions projected upon a plane. . This fact would materially alter the physical appearance of many of these objects. For example, the writer believes that the coronal arches existing above many of the prominence groups are in effect hemispherical shells of coronal matter. These shells are ejected by the stupendous eruptive forces residing in or beneath the prominences over which in most instances they are suspended.

In the case of multiple concentric arches it is likely that these eruptive forces are intermittent and possibly of decreasing magnitude. In this respect the formative action in relation to the coronal arches would be exemplified in the intermittent eruptions of certain terrestrial geysers. In this analogy, while the eruptive materials would be different and on an infinitely lesser scale in the case of the geysers, the procedure of the eruptive forces would be much the same. If the geyser discharge were in a less dense medium than our own atmosphere, one comparable with the solar surroundings, and if that atmosphere were in a comparatively quiescent state, the masses of vapors emitted undoubtedly would assume domelike aspects. If these hypothetical eruptions could be viewed from a distance in a horizontal plane, they would probably present an appearance in form similar to the solar coronal arches.

Also, many of the coronal features, especially of the ray or the striated type of emissions, may be apparently intensified by projection upon others lying in the same line of sight.

In some cases vacancies or gaps appear to exist in the general regularity of the coronal emissions. These gaps may be due simply to the lack of coronal matter, or they may depend upon the obscuration of detail by matter of a dark or to some extent of an opaque nature.

Instances of this latter class have been discussed at this and preceding eclipses. There seems to be sufficient evidence to confirm the existence of these light absorptive masses upon several of these occasions. It is possible indeed, that more of these opaque masses exist, in the coronal structure than had been heretofore recognized. When we consider that a bright ray of coronal matter projected upon a dark one will break up or obscure the latter, we can realize that the chances of recognizing dark objects of this class in the Sun's corona are not often presented.

An explanation for the existence of these dark masses might be found in the return of ejected coronal matter to or towards the chromosphere. This ejected matter having been reduced to a lower temperature, both by expansion, if gaseous, and by radiation in any case, may then assume comparatively dark or absorptive characteristics. It is difficult to realize, however, how much contrast could be 
produced even by temperature differences, as above, in matter so relatively near a radiating light source such as the Sun.

This conception is only tentative, as no absolute motion has been detected as yet in the corona at any single eclipse. At nearly every total eclipse conspicuous coronal disturbances are observed. These are generally on a considerable scale, and often exhibit a great amount of detail and contrast, especially on coronal photographs taken with the long focus instruments. It is in these inner regions of apparent violent disturbance, therefore, that variations, either in form or intensity, or both, are likely to occur when observed from widely separated stations.

With the 7.5-inch lens of 65 feet focal length employed by the Naval Observatory at the eclipse stations of Porta Coeli, Spain, 1905, and at the present eclipse, a degree of arc at the focal plane is equal to about 13.66 inches. Consequently one minute of arc would be equal to nearly a quarter of an inch, and one second to about 0.04 of an inch, respectively. This latter quantity is practically equal to a millimeter. Now the linear value of a second of arc at the distance of the Sun from the Earth is equivalent to approximately 450 miles.

It is therefore evident that changes on a stupendous scale must take place in such coronal disturbances before they can be detected upon the photographs produced with even this large scale apparatus. There are, of course, errors involved in these processes of observation, both atmospheric, instrumental, and physical, which augment the difficulties of detecting any coronal changes by obscuring the finer details. The size of the grain of the photographic plate and contrasts in the image are in part factors. These conditions, together with the nebulous character of the coronal markings in the present and previous disturbances, render the chances of detecting changes of form or brightness in these objects rather remote, even though the stations of comparison be widely separated. However, it can be stated with considerable confidence, that no very rapid changes of coronal form take place in the masses as a whole, even in the most favorable cases which have been studied up to the present time.

The polar rays.-These rays in the vicinity of the Sun's southern pole will be first described, conforming in arrangement to the discussion of the prominences. A description of the northern polar rays will then follow. For this purpose Negatives 2 and 4 made with the 65 -foot camera are available for the inner corona, the latter negative showing extreme extensions to a distance of about one-half of a solar radius.

Some reference will also be made to the photographs taken with the 6-inch visual lens with color screen. On account of the obscuration of many of the finer details in the outer extensions on all of these negatives by the thin cloud haze prevailing at the station, only a more general description of the corona can be furnished than at several of the previous eclipses observed by the Naval Observatory parties.

The south polar coronal region.-The polar rays of the inner corona at the Sun's southern pole present an unusual appearance. To the east of the pole the typical striated rays are present. These rays show no marked characteristics except in their anomalous inclination. Instead of a general tendency to curve away from the 
pole as they recede from the Sun, the rays here specified, near the Sun's axis, lean slightly towards the pole. At a position about $25^{\circ}$ to the east of this southern pole as a center, the rays on either side of this location for a distance of about $20^{\circ}$ have the typical appearance of the ordinary polar rays as usually observed. On either side of this specified center, the polar rays are seen to be of the ordinary striated type. They are, moreover, seen to curve symmetrically away from this center as rays of the polar type curve away from the Sun's axis. From what follows in regard to the description of the rays on the western side of the pole, it appears as though the pole of emission of the typical polar rays had been shifted about $25^{\circ}$ towards the east from its usual position, at this eclipse. The polar rays are often unsymmetrically distributed to some extent, as observed in previous eclipses, but seldom in so marked a manner as upon this occasion, and never to the writer's knowledge where the cause of such displacement is so apparent as in the present case.

On the western side of the Sun's southern pole the coronal rays are much more complex. This is evidently owing to the unusual conditions prevailing in this region. Under the description and position of the prominences the remarkable group of these objects situated in this south polar region is fully discussed. The position of this group extends from P. A. $164^{\circ} .0$ to $183^{\circ} .0$ from latitude $-86^{\circ} .5$ on the east side of the pole to latitude $-74^{\circ} .5$ on the western side. In the region specified above the corona surmounting this prominence section is seen to consist of coronal arches above the larger prominence features. Two of these arches can be distinctly traced, surmounting the features mentioned. In this same region straight, or nearly straight, emanations are also seen. These, instead of individual rays, as observed upon the eastern side of the southern pole, consist of several strong wings of parallel rays. The rays composing these wings are striated, and appear to be of the true polar type. In this same general region coronal matter in a chaotic mass is apparently mixed with the features just described. As a whole, this western side of the Sun's southern pole exhibits an extreme amount of solar activity.

To the region west of this large prominence sector at the southern pole and extending approximately to the prominence at P. A. $204^{\circ} .0$ to $206^{\circ} .5$ (Lat. $53^{\circ} .5$ to $\left.-51^{\circ} .0\right)$ attention is now directed. In this region three broad and nearly concentric bands of coronal matter are observed curving towards the equatorial regions. These bands are the southern bases of a magnificent series of concentric coronal arches spanning the prominence above specified. The entire region involved is also filled with diffused coronal matter. The regions heretofore described generally are occupied by the typical polar rays of the striated type in solar eclipses previously observed. The prominence groups near this pole have therefore radically altered the character of the type of coronal forms usually to be found existing in these regions. These groups of prominences have influenced the aspect of the corona where they principally are seen-to the west of the southern pole. In these polar prominence regions the types of coronal detail generally found at lower solar latitudes exist. On the eastern side of the pole, however, where no prominence formations occur, the corona exhibits rays of the usual polar type.

It therefore seems likely, from the study of this and former eclipses, that prominence formations are the main factors in the modification of coronal detail. 
This statement is especially probable regarding the conditions obtaining in the polar regions at the present eclipse.

The north polar coronal region.-At the Sun's northern pole we also find a displacement of the typical striated polar rays. Here, as in the south polar regions, there exists a prominence group in close proximity to the pole. This group is described at P. A. $345^{\circ} .0$ in this report under the discussion of the prominences. A tendency towards an arch formation is seen in the corona above this group. This arch effect, however, is rather faint, especially at its upper limits, and is not a prominent coronal feature.

The general form of the coronal detail in this vicinity is observed to consist of numerous tapering wings of considerable brightness for polar emanations. These wings are of comparatively great breadth in proportion to their visible length; as seen on the photographs of the inner corona. The line of separation or demarkation between these polar rays or wings and that of the equatorial streamers on the western side of the north pole is not very well defined. This is on account of an enormous coronal disturbance, together with a magnificent complex system of arches above the general region of the immense prominence group at P. A. $296^{\circ} .0$ to $303^{\circ} .0$.

A fair estimate of the arc of the sector on the western side of the Sun's north pole occupied by these polar rays as a distinctive mass, though mostly of the wing type, is about $20^{\circ}$.

For a distance on the limb of about $15^{\circ}$ on the eastern side of the prominences near the north pole the polar rays are of the same general character, or wing variety, as those existing on the western side of this pole. Beyond the above region, to the east for about $33^{\circ}$ and terminating at a distance of $48^{\circ}$ from the northern pole, the coronal rays are of the striated type. Throughout this entire region these rays have a tendency to lean slightly from the perpendicular in the polar direction. With a few exceptions they are nearly straight. There is, however, an irregularity of structure at a distance of $35^{\circ}$ to $40^{\circ}$ from the north pole in P. A. $22^{\circ} .5$ to $27^{\circ} .5$. In this region it appears that while the regular striated structure exists, there is, in addition, a disturbed section in this vicinity. At the above position angles, somewhat distant from the Sun's northern pole, the development of coronal irregularities might be expected.

Equatorial regions.-The equatorial regions of the inner corona on the Sun's eastern limb will be first described, following which a description of those on the western side will be given. The equatorial coronal features on the eastern side of the Sun exhibit much less general activity and detail than those upon the western side. This fact is undoubtedly due to the great contrast in eruptive forces as exhibited by the large prominence groups in these different locations. On the eastern side of the Sun in the equatorial regions there is visible but one of these conspicuously large prominence masses. This is described in detail under the discussion of the prominences. This large prominence group specified above is situated at P. A. $49^{\circ} .5$ to $55^{\circ} .5$. The inner corona above this region and for a considerable distance on either side shows evidence of violent disturbances of a complex nature. 
The limit of the northern polar rays at this eclipse, judging from the physical aspect on the eastern side of the Sun, is in latitude $+39^{\circ} .5$ at P. A. $38^{\circ}$. At this position angle and having a width of about one degree a conspicuous dark gap exists in the corona. The edges of this gap are fairly well defined throughout its course in the inner corona. The gap is slightly inclined northward near the Sun but shows a slight curvature in the equatorial direction as it recedes.

This gap increases in absolute width as the distance from the Sun increases. The coronal matter on the southern side of this gap is evidently influenced by the conditions existing to the south of it, in the region above the large prominence at P. A. $49^{\circ} .5$ to $55^{\circ} .5$ already mentioned. The coronal conditions referred to consist of a magnificent system of coronal arches suspended above this prominence. These arches, while plainly visible as such, are involved in coronal matter exhibiting considerable agitation. This is especially the case in the area lying within the lower arch and over the large prominence itself. Here an irregular column of coronal matter is seen, apparently having origin near the summit of the spiral part of this prominence group. The arch system above these large prominences consists of three distinct members. The lower two, while normally separated on the equatorial side, appear to blend on the northern end, thus presenting a very unusual aspect. The southern end of the third, or upper, arch is irregular, showing a bend outward a short distance above the limb. It then returns to its normal course again for the remainder of the arch.

On either side of this arch system the coronal rays in the immediate vicinity appear to be subject to the influence of this arch system. There is a tendency to. curvature in these outlying rays towards the arches, especially in those on the northern side as they recede from the Sun. The entire region just described, extending from the north polar rays to the vicinity of the solar equator, shows the dominating influences over the features of the inner corona of this large triangular group of prominences.

Beyond the solar equator, to the south, and extending to the region of the southern polar rays the character of the inner corona on the Sun's eastern side is quite different from that of the northern latitudes just described. In general, this. is a sector on the Sun's limb where many but no large prominences are observed and where the serrated-edge appearance in the chromosphere has been noted in the text (see discussion of the prominences from P. A. $71^{\circ}$ to $132^{\circ}$ ).

While the extent and brilliancy of the coronal rays of the inner corona on Negatives 2 and 4 with the 65 -foot instrument here exceeds that of the northern hemisphere on the Sun's eastern side, the character of the detail observed in the corona of this southeastern sector is quite different from the forme: type of detail in this region in south latitude, lying between the equator and the southern polar rays, is mostly of wing formation. There is considerable variation in the direction of many of these wings from a true vertical as they recede from the Sun. This variation of direction of emission is especially noticeable near the equator. Just to the north of the equator and emanating from a position in close proximity to the base of the coronal arch previously described a comparatively bright, straight ray is emitted. As we pass to the southern hemisphere on this. $101141^{\circ}-26-$ PT II- -62 
same eastern side, and covering a sector of about $14^{\circ}$, the structure of the inner coronal rays consists of broad and comparatively bright wings. These have various inclinations towards the south and are interspersed with matter of no decided structure. Those to the south cross the winglike emanations next to be described. These latter exist above the region occupied by a series of small prominence groups between $\mathrm{P}$. A. $89^{\circ}$ and $119^{\circ}$. In this sector the corona is comparatively bright. It exhibits here considerable detail, being mostly composed of narrow wings. These wings have, on the whole, an inclination from the vertical in the direction of the equator.

This inclination, though slight, is especially pronounced in the equatorial regions, somewhat diminishing in the wings of the higher southern latitudes. Intermingled with these inclined wings, other rays, somewhat fainter, are seen taking a perpendicular course as they recede from the Sun. The coronal rays, as a whole, are less intense and extensive on the Sun's eastern side at this eclipse than on the western side.

The southern polar rays which appear next in order have already been described.

The inner corona on the Sun's western side will now be analyzed.

Beginning in the southwest quadrant, next to the polar rays, there exist series of arches in the corona, above the prominence group at P. A. $204^{\circ} .0$ to $206^{\circ} .5$. These arches have already been mentioned in connection with the southern polar rays, to which they are adjacent. They cover a span in an equatorial direction extending nearly to the feature next to be described.

The region above referred to is situated in the sector of the prominences at P. A. $225^{\circ} .5$ to $234^{\circ} .0$. The corona in this locality exhibits considerable detail, mostly of the striated or narrow wing type. These features, while showing some deflection, in general take a nearly perpendicular course outward from the Sun. This region is intermediary, and lies between the bases of the enormous arch system previously described nearer the southern pole, and that one which will be described later, in the vicinity of the equator.

At P. A. $236^{\circ} .5$ and in this immediate neighborhood an intense coronal disturbance is seen. This object, though not of great magnitude, exhibits considerable detail and contrast. It is of great comparative brightness, as seen on the shorter exposure negatives with the long focus instrument. The coronal matter is intimately involved in the prominences of this region. Some of this prominence matter is of such tenuity that it is difficult to differentiate it from the bright coronal matter adjacent to it. This region, as noted previously, was studied in comparison with photographs made at other stations for coronal changes. This disturbed coronal region offered the most promising possibilities for such investigation, on account of the contrast and unusual detail to be found in such a comparatively restricted area. No changes could be detected, however, in either its form or brightness with the material and data available. It may be stated, also, that this coronal disturbance lies close to the southern edge of the outer arch of the concentric series spanning the immense prominence group following. In fact, as the coronal matter extends outward from the Sun at this disturbance it appears to acquire a curvature in the same general direction as the outer arch of the series above mentioned. 
The next feature of the inner corona on the Sun's western side is the system of magnificent arches spanning the enormous "fantastic" prominence group at P. A. $249^{\circ} .0$ to $264^{\circ} .5$. This arch system at its base covers a sector beginning near the limit of the coronal disturbance at P. A. $234^{\circ} .0$ and extends to P. A. about $285^{\circ} .0$. The arches of this system, especially those of the parts nearer the solar surface, are not as well defined, and are not spaced at such regular concentric intervals, as in most of the phenomena of this class previously described, in connection with this eclipse. These arches, moreover, indicate a much greater expulsive force than those observed in any of the preceding cases.

The fact that the prominence over which this arch system is located is in a state of unusually violent change would indicate that its explosive forces had a direct connection in the magnitude, brilliancy, and extent of this coronal upheaval. In connection also with the fact that it is the only prominence of large proportions in the sector described it is evident that the coronal masses above it are induced and influenced by its activity. It should be stated, however, that an intense coronal emission is seen above the low bank of prominences to the south of this large eruptive prominence, over which the arch system is located. The coronal matter above this prominence bank appears to be composed of matter of irregular texture, and may simply be the intensified bases of some of the arches of the system spanning the large eruptive prominence. Succeeding the arch system above discussed is a conspicuous gap in the coronal emission. This gap is more apparent both in width and intensity as the distance from the Sun increases. It is evidently occasioned by the coronal rays being deflected as they recede from the Sun by the arch system already described and also by the influence of the coronal emissions next to be discussed.

The region on the Sun's western side, now to be described, extends from about P. A. $290^{\circ} .0$ to the northern polar rays. It includes a sector approximately of $47^{\circ}$ ending at P. A. about $337^{\circ}$. The coronal emission in this sector is of about the same order, both in brightness and extent, as in the sector of the arch system preceding it. This region overlies the immense prominence groups extending between P. A. $291^{\circ} .5$ to $303^{\circ} .0$. These groups of prominences while extensive are more massive, or of a greater density, in general, than the "fantastic" prominence. They do not exhibit the detailed structure, as a whole, which was noted in the former case.

The corona in the sector above these great prominence groups is much disturbed and exhibits considerable detail. The detail, as a whole, is much confused and of a very irregular structure. Upon analysis, it is evident that this irregularity is due in part to the fact that in this region numerous and comparatively small coronal arches exist. These arches are distributed in a heterogeneous manner. Their partial projection upon each other, in many instances, produces the mottled appearances observed in this region. There is also a considerable amount of coronal matter involved either in a state of local diffusion or of the smaller wing type. This matter apparently is repelled in a perpendicular direction from its source.

The region just described in the northern hemisphere on the Sun's western side, together with the magnificent arch system and other features previously analyzed in the southern hemisphere on this same side, as the coronal matter is traced out- 
wards, is seen as two enormous wings. These wings and their arrangement are in evidence on the exposures made with the 6 -inch visual lens and color screen.

On the Sun's eastern side in the same manner but a single coronal extension is thus observed. These wings, or extensions, as above, gave the "heart shaped" appearance in the form of the corona as observed visually at totality.

The outer corona at this eclipse can not be satisfactorily discussed from our negatives, since the cloud haze prevailing at the station during totality cut down the contrast.

The observed manifestations of activity in the inner coronal regions, in conjunction with the many active features of the chromosphere, and prominences marks this eclipse as one of the most instructive. As each total solar eclipse is observed, it is found that the features of the solar surroundings are subject to great changes from one eclipse to another one. It is realized that this variation depends upon the solar activity, as visualized in the prevalence of solar spots, faculæ, and prominences. This is demonstrated by the fact that the general type of corona can be predicted in advance with considerable certainty from the position of the eclipse in the sun-spot cycle. Until it becomes possible to study the corona without a total eclipse, the present efforts of different scientific institutions to observe these events systematically should be continued.

The chromosphere and prominences.-The prominences observed at this eclipse on the negatives made with the 65-foot camera were numerous. They were distributed about the disk of the Sun with considerable regularity. One of the remarkable features of this nature was the enormous prominence group existing at and near the Sun's southern pole. This unusual prominence group extends over an arc of $18^{\circ}$. As seen projecting above the Moon's limb, the large prominence feature at the eastern end of this group is located in line with the Sun's axis. This prominence compares favorably in brilliancy and size with the other objects of this class visible at this eclipse.

The northern pole has likewise a fairly large prominence near it. These unusual conditions at the poles have had a marked effect upon the character of the polar rays, as will be described later.

Three other prominence groups of large size are also observed upon these negatives. One of these groups is located on the western side of the Sun, near the solar equator. The other two are situated in the northern hemisphere, on the eastern and western limbs, respectively. With the exception of the one near the equator these latter prominences are normal features. They are situated at about the upper limits of the sun-spot zones, where prominences are most frequently observed. In the southern sun-spot zone on the eastern limb several prominences form an irregular group extending nearly to the equator.

On Negative 6, the last exposure of the series with the 65-foot instrument, the chromosphere is seen projecting above the Moon's limb, over an are of about $45^{\circ}$. Several slight interruptions to this continuity occur near the southern limit of this arc, caused by irregularities on the Moon's limb. A medium-sized and a large flattopped prominence are observed at this locality. These are bright objects and appear to be but a general elevation of the chromosphere in this region. A serrated 
appearance of the chromosphere is seen here. It is similar to that observed on Negative 1, showing the opposite chromospheric limb. These serrations, however, have not the same degree of regularity as those shown on the opposite side, on Negative 6.

The two largest prominence groups visible at this eclipse are located on this western chromospheric region. Each is of peculiar form and structure. A study of their sources of origin, as they emerge from the chromosphere, will be possible from the photographs.

The chromosphere is not shown upon any of our negatives in the southern polar regions. If available, these would have enabled a better study to have been made of the unusual conditions existing in that locality. The station at Baker was very close to the center of the Moon's shadow path. The conditions with respect to the visibility of these polar regions were consequently known in advance. Where the Naval Observatory has been able to equip and send out more than a single party, one of these has generally been placed just inside the northern or southern limits, of the shadow.

Located in the same general regions, often apparently in close proximity, prominences are seen varying considerably in intensity. There are two reasonable methods of discussing these objects and this process is often necessary in connection with the corona.

In the first hypothesis we may consider that they are all situated upon the solar limb but vary in relative brightness. In the second instance we may assume, that while of the same degree of brilliancy, or nearly so, they are located at different distances from the Sun's apparent edge. This latter hypothesis, of course, is the more reasonable.

The strong absorption of the solar rays by the Sun's atmospheric envelope is a well-known fact, both from photographic and visual observations.

The light emitted by the prominences would thus suffer a varying degree of absorption depending upon their distances from the solar limb. Consequently, on the average, the more brilliant objects of this class would thus be situated on the sphere in front of the Sun's limb. Likewise, those of average intensity would be located at or near the limb. The fainter prominences would consequently originate in the regions beyond the limb of the Sun.

The height of these prominence masses would have a direct bearing upon the degree of absorption, depending upon the elevation of the solar absorptive media and the rate of rarefication with altitudes. Moreover, where the prominences are located either in front of or behind the limb, the origin of the base at or near the chromosphere is occulted. This effect may be partially obscured by the interposition of the Moon's limb, except where the projecting chromosphere is visible.

It may therefore be regarded as feasible, with a considerahle accuracy, for an experienced person to diagnose the relative positions of the prominences with respect to the Sun's limb. This may be done in many cases by the general appearance of the prominence images on the eclipse photographs. The consideration of the surrounding coronal detail also has a bearing upon these conclusions. 
The prominences.-As usual the prominences will be described in order of their position angles. These angles will be counted from the north point of the heavens, towards the east. They will extend from P. A. $0^{\circ}$ about the solar circumference to P. A: $360^{\circ}$.

In order that the solar features may be described as a whole, the arc beginning at P. A. $355^{\circ} .5$ will be taken as a position of departure.

P. A. $355^{\circ} .5$ In this region covering an angle of $36^{\circ}$ and to the eastward of the to $31^{\circ} .5$. Sun's northern axis, the chromosphere is hidden by the Moon upon Lat. $+82^{\circ} .0$ to $+46^{\circ} .0$ all of the negatives. This sector is practically devoid of prominences. Slight indications of several small prominences are in evidence, but these are of no especial moment.

P. A. $32^{\circ} .0$ to $49^{\circ} .5$

Lat. $+45^{\circ} .5$ to $+28^{\circ} .0$

P. A. $49^{\circ} .5$ to $55^{\circ} .5$

Lat. $+28^{\circ} .0$ to $+22^{\circ} .0$

The chromosphere is also hidden by the Moon to some extent in this region on the negatives. Rising above the Moon's limb, a series of small prominences is visible in this sector. These have a general similarity in appearance to each other, but are not of importance. A somewhat larger prominence, of considerable brightness, occurs. at P. A. $32^{\circ} .0$.

One of the largest and brightest prominence groups visible at this eclipse is situated at this location. This group attains an altitude of approximately $120^{\prime \prime}$ above the Moon's limb, which on Negative 1 just. covers the chromosphere in this locality. As a whole this prominence group is roughly triangular, with a small wing-like expansion at its summit. Upon our negatives it is seen to be composite, being built up of three different principal emanations.

The first of these may be described as four or more individual flames closely associated together. These flames rise to different elevations, in nearly a vertical direction, the northernmost being the highest. The base of this triangular group occupies an arc of about $2^{\circ}$ upon the sector taken up by the entire prominence formation. To the north of the triangular part of this group a long thin filament of prominence matter is seen. This filament, slightly arching, leans towards the triangular formation. Beneath this filament, near its base, two other individual formations are seen. The first of these objects curves towards the south, while the other is in the form of a small Y-shaped prominence. Both of the objects just mentioned are interrupted at their bases. They have the appearance of being sharply screened off by some light absorptive or opaque matter beneath them. In outline, the northern part of the composite triangular prominence is roughly pyramidal. It is composed of two sections, each of which presents considerable detail. No evidence of wings or extensions, however, are seen projecting from this part of the prominence. The most interesting feature of this composite group is a tall spiral prominence. The base of this feature is occulted by the southern part of the triangular group as first described as a whole. This spiral column is located in a gap between the other 
two components of the group. About one fourth of its elevation surmounts the pyramidal feature of this aggregation.

The convolutions of this spiral column are extremely regular. Judging from its appearance upon the negatives, it must have a rotary motion about an axis.

At its summit this spiral column expands into two short pointed arms or wings. This winged aspect of the spiral, and the composite group of prominences which it surmounts, give to this group with which it is associated the appearance of the so-called "eagle" type of prominence.

The spiral column leans slightly to the north as it ascends. It is one of the unique and interesting features of this eclipse. In another place in this report the magnificent coronal arch system spanning this group of prominences will be described.

The definition of these objects as seen on Negative 1 is extremely fine. The slight cloud-haze veiling the sky in the vicinity of the eclipsed Sun, by screening down the extreme photographic brilliancy of the prominence, has apparently increased the usual amount of detail visible on these negatives. Part of this unusual definition on these short exposures was undoubtedly due to the remarkably good seeing conditions at totality.

P. A. $56^{\circ} .0$ In this region the continuity of the projecting chromosphere is to $70^{\circ} .5$ considerably broken up by the irregularities of the Moon's limb. A series of short narrow prominences varying somewhat in size is visible in this sector. None of these objects are of any considerable magnitude. With the exception of a $\mathrm{Y}$-shaped prominence at P. A. $63^{\circ} .0$, they project from the Sun directly upwards.

P. A. $71^{\circ} .0$ In this equatorial region the chromosphere presents but slight to $80^{\circ} .0$ irregularities. Projecting from the chromosphere are numerous small Lat. $+6^{\circ} .5$ to $-2^{\circ} .5$ needlelike formations, as seen on Negative 1. These have, from their brilliancy, a striking resemblance to prominence matter but are of unusual type. These formations are all inclined to the south as they recede from the Sun, at angles of from $30^{\circ}$ to $40^{\circ}$ from the perpendicular. In some respects these needlelike projections resemble the bases of bright coronal rays. They are much more intense, however, as but a slight trace of the general coronal envelope is seen upon this negative.

A slight break exists in the continuity of the chromosphere on Negative 1 at P. A. $71^{\circ} .0$, as if it were here interrupted by an elevation upon the Moon's limb. On Negative 2, where the chromosphere is covered by the Moon in this region, five of these bright narrow inclined prominences are still visible. 
P. A. $80^{\circ} .5$ This is the position of an arching prominence of medium elevato $82^{\circ} .5$ tion and average brightness. A small prominence formation is seen Lat. $-3^{\circ} .0$ under and near the middle of this arching prominence. With this to $-5^{\circ} .0$ exception the space under the arch is unobstructed by other prominence matter.

P. A. $83^{\circ} .0$ In this locality a medium flat-topped prominence of considerable to $85^{\circ} .5$ brilliancy exists. It has a projection to the south from its upper Lat. $-5^{\circ} .5$ levels, arching downwards over a small prominence of slight elevation.
to $-8^{\circ} .0$

P. A. $85^{\circ} .5$ The chromosphere is devoid of prominence in this region, but to $89^{\circ} .0$ presents a finely serrated profile.

Lat. $-8^{\circ} .0$ to $-11^{\circ} .5$

P. A. $92^{\circ} .5$

This is a series of low lying prominences. They rest upon three to $97^{\circ} .0$ short bases or supports between them and the chromosphere, which Lat. - $15^{\circ} .0$ are spaced at nearly equal intervals. These bases are also connected - to $-19^{\circ} .5$ by irregular tenuous filaments of prominence matter. The northern end of the upper arch of the next following prominence group rests upon the middle parts of the upper regions of the prominence group here described.

P. A. $100^{\circ} .5$ The brighter parts of this prominence have an elevation of about to $102^{\circ} .513^{\prime \prime}$ above the chromosphere. Extending from the summit of the Lat. - $23^{\circ} .0$ prominence at this elevation is a long curved branch of greater to $-25^{\circ} .0$ tenuity leaning towards the north. Although faint in its upper levels, this branch can be traced as an irregular arch, extending to the prominence preceding, where it already has been mentioned. Beneath this arch feature just described is another brighter arch, concentric with the one above it. This lower prominence arch is brightest near the middle. The chromosphere beneath these arch formations, though undulatory, exhibits no evidence of extreme agitation.

P. A. $119^{\circ} .0$ This position angle designates the location of a small and very Lat. $-41^{\circ} .5$ faint prominence. Closely adjoining this feature, to the south is a bright, slender, triangular, or spike-shaped, prominence. This latter object is of small dimensions, and is followed by others of the same type, between it and the brushlike prominences next mentioned.

P. A. $126^{\circ} .5$ A series of pointed prominences of slight elevation, having a to $130^{\circ} .0$ brushlike appearance, is seen in this sector. The pointed rays at Lat. $-49^{\circ} .0$ the eminence of these chromospheric elevations have a general tendto $-52^{\circ} .5$ ency to lean towards the south. From their general appearance in Negative 1, these rays have the aspect of being the roots, or bases, of the coronal rays; although the direct connection can not be established with certainty. It may be stated, however, that from the region where the chromosphere becomes visible, on Negative 1, to the above locality the inner coronal rays have a finely striated appearance. In the aggregate these rays by combination form the short wings observed in the inner corona in this region. 
P. A. $132^{\circ} .0 \quad$ A small wedge or triangular-shaped elevation is here seen rising Lat. $-54^{\circ} .5$ above the chromosphere. Between this location and P. A. $139^{\circ} .0$ the chromosphere is characterized by objects of this description, though smaller. As in the preceding case, these specified objects lean in a southern or polar direction. As seen in this region on Negative 4 , it should be noted that the inner coronal rays have also an inclination in the same direction. This fact is especially noticeable close to the Moon's limb.

P. A. $143^{\circ} .5$

A faint dome-shaped prominence showing above the Moon's

Lat. $-66^{\circ} .0$ limb is visible at this position. As thus seen it has a width of about half a degree and a corresponding linear elevation. From the faintness of this object it is likely to be the top of a prominence situated beyond the edge of the Sun's limb.

P. A. $161^{\circ} .0 \quad$ No large prominences are visible in this region. At this position Lat. $-83^{\circ} .5$ angle, or $6^{\circ} .5$ to the east of the southern pole, a faint low prominence is visible. On Negative 1 the chromosphere is covered by the Moon's disk from this position angle to P. A. $245^{\circ} .0$.

P. A. $164^{\circ} .0$ This is the position of a large prominence which is part of a to $168^{\circ} .0$ group covering the region of the Sun's southern pole. In its relation Lat. on E. side $-86^{\circ} .5$ to to the Sun's axis of rotation this group of prominences is unique. Lat. on W. side So far as the writer is aware, at no previous eclipse have large masses of Pole $-89^{\circ} .5$ of prominence matter been thus situated. The forms or type of prominences found in this group are not unusual. They have in general a similarity of appearance to other prominence types found in lower latitudes. A low prominence of about $10^{\prime \prime}$ elevation, forming part of the larger structure, lies at the southern pole. At least it is seen in the line of sight with the Sun's axis. The greatest elevation in this group is $40^{\prime \prime}$ above the Moon's limb. The true elevation above the chromosphere would be about $10^{\prime \prime}$ greater, allowing for the covering by the Moon. This highest part is brighter than the other prominences of this group, and is nearly rectangular in outline. It is seen to be composed of several tongues of flame. Surmounting its summit is a small pinnacle of flame. In form, though of lesser size, this feature resembles the largest prominence observed at the eclipse of May 28, 1900.

On Negative 1, where this prominence is seen nearly to the chromosphere it has the appearance of two of these prominence objects superposed. As interpreted both by difference in type and contrast in brightness, one of these prominences should be located partially behind the other. The fainter part of the prominence apparently acts as a background for the brighter one. In outline this fainter part appears as an arching prominence, a type which is quite prevalent at this eclipse. Where it reappears from behind the brighter intervening prominence (on Negative 1), it again comes in contact with the Moon's limb, after which an upward turn is noted. The 
fainter prominence parts, the background portions above referred to, have a rolling cloudlike aspect, as if agitated by strong convection currents.

Referring to the brighter prominence of this group again, it appears from Negative 1 to be constituted of two principal tongues of flame extending to an elevation of about $40^{\prime \prime}$ above the Moon's limb. These parts are joined together by a diagonal connection at about mid-elevation. On Negative 2, on account of the longer exposure, these conditions are not recognized, owing to the blending of the features above detailed. The brighter and fainter parts of these prominences are apparently combined on this latter negative and are shown, but with little contrast, between the different features. The appearances as given in the first part of the description in relation to this group are consequently not shown on Negative 2. These different aspects from Negatives 1 and 2, taken in rapid succession, are not due to structural changes occurring in the shorttime interval between the exposures. They are due to limitations of the photographic processes, in connection with bright objects presenting considerable contrasts, and to differences of exposure. The appearance at this eclipse of this unusual polar group of prominences seemed worthy of an extended description. This is especially the case when referring to the discussion of the inner corona. We find that the polar rays of this region are greatly and uniquely disturbed. This disturbance can be traced to the presence of the prominence groups about the Sun's southern pole. It should be realized, however, that many of the features described are visible only upon the original negatives or upon glass positives from the same. A certain amount of detail is necessarily lost in the reproductions for the volume on account of the processes employed.

P. A. $173^{\circ} .0$ This prominence is of the "rose" or flower type. It has a Lat. $-84^{\circ} .5$ width of about $2^{\circ}$ on the Moon's limb, with an elevation of $15^{\prime \prime}$. Its location is about midway between a group of three prominences to be mentioned later and the large prominence group near the southern pole, just described. The entire region between P. A. $173^{\circ} .0$ and $177^{\circ} .0$ shows indications throughout of intense prominence activity.

P. A. $175^{\circ} .0$ This is a wide prominence of slight elevation. The top of this Lat. $-82^{\circ} .5$ prominence is arching, but with considerable irregularity. It is not a brilliant object. This prominence fills in the sector between the triple group next described and the prominence previously mentioned.

P. A. $177^{\circ} .0$ In this series of three bright prominences on the western side of to $183^{\circ} .0$ the southern pole the brighter parts are elevated about $26^{\prime \prime}$ above the Lat. $-80^{\circ} .5$ Moon's limb. The variation of individual intensity among the objects to $-74^{\circ} .5$ of this group is quite noticeable. The first object of this prominence group is somewhat larger than either of its associates. It is also 
somewhat fainter and of thinner texture. It is of crescent form, with the horns inclined towards the south.

The second prominence of this group is of great intrinsic brightness. It is projected upon a background of much fainter prominence matter. Appearances would therefore indicate that the brighter of these prominence masses must be situated in front of the others as we observe them. This brighter prominence is multiple. It is composed of a short central elevation with a smaller companion, close and to the north of it. On either side of these, two somewhat larger prominences are seen, bending over towards the center of this group.

The last of this series of prominences presents no unusual features. It is built up of several distinct flames. These are inclined from the perpendicular towards the north. A thin filament of prominence matter is seen extending from the summit of this group. This filament is in the form of a large loop, extending nearly to the middle of the region embraced by this prominence group. In the later photographs with the 65-foot instrument many changes are noted in the structure and shape of this filament. On these latter exposures several additional threadlike forms of considerable irregularity are visible in this region.

A study of the several negatives would indicate that with the - exception of the second or brighter prominence, here described, the other prominences of this sector, covering $6^{\circ}$ of arc, form a continuous associated group.

P. A. $183^{\circ} .0 \quad$ Between these position angles there are no prominences visible to $204^{\circ} .0$ upon any of our negatives.

Lat. $-74^{\circ} .5$

to $-53^{\circ} .5$

P. A. $204^{\circ} .0$ to $206^{\circ} .5$ considerably greater elevation than the others. Its height above the

Lat. $-53^{\circ} .5$ Moon's limb is $23^{\prime \prime}$. The prominence to the south of the preceding to $-51^{\circ} .0$ is much shorter, being of but about $6^{\prime \prime}$ above the limb of the Moon. The third prominence of this series is close to and north of the middle one of this group. It projects about $18^{\prime \prime}$ above the Moon's limb. This prominence is separated from the middle one by a narrow clear space. Filaments of faint prominence matter appear to connect the upper elevations of these three prominences. This matter is somewhat irregular and fragmentary. It shows a tendency towards an arching formation as it spans this region. It may be here stated that a conspicuous set of coronal arches also exists in this region, having the above prominence group as a center of curvature.

P. A. $206^{\circ} .5$ No indications of prominence formations are seen between these to $214^{\circ} .5$ two position angles, although the Moon covers the chromospheric Lat. $-51^{\circ} .0$ limb in this region to some extent.
to $-43^{\circ} .0$ 
P. A. $214^{\circ} .5$ This region contains a group of small prominences of slight imto $218^{\circ} .5$ portance. Those at either end of the sector are relatively bright, Lat. $-43^{\circ} .0$ though rising but little above the Moon's limb. Several prominences to $-39^{\circ} .0$ of greater elevation are seen between the two end members of this P. A. $225^{\circ} .5$ group.

to $234^{\circ} .0$ Between P. A. $218^{\circ} .5$ and P. A. $225^{\circ} .5$ no prominences are Lat. $-32^{\circ} .0$ visible, but in the region now discussed a series of small promito $-23^{\circ} .5$ nences show above the Moon's limb. With the exception of a pair of prominences at P. A. $229^{\circ} .0$, they present no special features. These latter prominences are connected by an arch of prominence matter somewhat less bright than themselves.

P. A. $236^{\circ} .5$ In this region is a series of three prominence groups, which, while to $245^{\circ} .0$ bright objects, vary somewhat in height and characteristics. Each Lat. $-21^{\circ} .0$ of these features will be described separately.

to $-12^{\circ} .5$ The first of the series is situated at P. A. $236^{\circ} .5$. The appearance of this prominence indicates considerable activity. It is quite a brilliant object, and is composed of several closely associated flames. Extending above this prominence and closely conforming to it in outline, is a much fainter area of prominence matter, or a highly condensed region of the corona. It is difficult in this locality to distinguish between the outlying characteristic materials of this prominence, and that of the bright coronal area above it. These features are of such varying intensity, both showing considerable detail, that they gradually blend together. On either side of this prominence, covering an arc of about $5^{\circ}$, as well as above it, is the most intense coronal disturbance in evidence at this eclipse. In another part of this report the features just described will be especially discussed as regards this coronal disturbance. An examination of this disturbed region was also made on photographs from several eclipse stations for possible changes in the corona. Between P. A. $237^{\circ} .5$ and P. A. $239^{\circ} .5$ in this sector a low prominence is seen. This prominence decreases slightly in elevation with increase of position angle. To the north of this region the chromosphere begins to appear from behind the Moon's limb on our last exposures, near the end of totality. The chromosphere is covered upon all of our plates from beyond the south polar regions to that now stated.

The concluding part of this sector, from P. A. $240^{\circ} .0$ to P. A. $245^{\circ} .0$, Lat. $-17^{\circ} .5$ to Lat. $-12^{\circ} .5$, is occupied by a bright bank of low prominences. These prominences can scarcely be distinguished from the chromosphere, except for their slightly greater elevation. 
P. A. $245^{\circ} .5$ The chromosphere in this region, to the north of the prominence to $249^{\circ} .0$ bank just described, is also in a quiescent state, and presents a very Lat. $-12^{\circ} .0$ similar appearance. At both of the position angles given, slight gaps exist in the continuity of the chromosphere. These gaps are undoubtedly owing to irregularities upon the Moon's limb. The quiescent state of these low prominence banks is extremely interesting. They lie between the previously described prominence surmounted by the intense coronal disturbance and the enormous "fantastic" group next to be described. The fact that this low prominence bank is beneath the southern part of the enormous system of coronal arches spanning this general region is also noteworthy.

P. A: $249^{\circ} .0$ Between these position angles, beginning at the low bank of to $264^{\circ} .5$ bright prominences previously described, this region contains the most Lat. $-8^{\circ} .5$
to $+7^{\circ} .0$ complex and "fantastic" prominence group visible at this eclipse. Taken as a whole, this group resembles in a rude way some enormous grotesque insect. On Negative 6 with the 65 -foot instrument this region is shown down to the chromosphere. Upon a careful examination of the original negatives this prominence group appears to be a very intricate object. As a whole, it can be analyzed as two fundamentally distinct groups, each of a separate type. The criterion of their separate identities lies in the form and distinct characteristics of the several parts, showing it to be a composite object. The different directions with respect to the vertical in which these various objects trend, and their sources of origin at the chromosphere, also indicate separate entities.

The most intense part of this composite group originates in a low-lying prominence, which is also probably a distinct feature in the northern part of this sector. The stem base of this intense part is fainter than the other portions of this prominence. Detail seen in this ascending column would indicate a cyclonic or rotary motion about an axis. After attaining an altitude of $20^{\prime \prime}$ above the chromosphere this column expands considerably and becomes brighter. The stem base of this object leans slightly towards the south. This inclination increases gradually with elevation. As a whole this prominence has an inclination of about $45^{\circ}$.

The expanded portion of this prominence also exhibits considerable detail. Much of this detail is evidently occasioned by the part of this prominence group to the south of the feature just described. This latter is apparently a distinct and independent formation in the vicinity of the inclined prominence already mentioned. This independent formation to the south consists of a group of filament prominences. These arise from the chromosphere and intersect the more intense inclined part described above. These filamentary prominences consist of three narrow and nearly vertical columns. 
Three other prominences of similar description are seen closely adjoining the ones just mentioned and to the south. These latter are less well defined and are somewhat broken into detached fragments as they ascend. They are also more tenuous than the others. The three first mentioned exhibit details in the ascending stem after leaving the chromosphere, as if subject to axial rotation. This vortex motion appears to be the prevalent condition in many of these objects at this eclipse.

Arriving at the height corresponding to that of the bright inclined prominence of this group, the filament prominences deviate towards the former. They then curve over abruptly in the same northerly direction, gradually falling back towards the Sun. In this recessional path they curve slightly, and can be traced back to the chromosphere beyond the inclined bright prominence of this "fantastic" group. These curved filaments form a nearly parallel series as they take the course described. The uppermost of these filaments extends over to the bright inclined prominence to be noted later, at P. A. $264^{\circ} .5$.

A peculiar condition seems to exist where the filament prominences cross the bright inclined prominence of this group. At the crossing the bright prominence suddenly becomes fainter. This brighter prominence is also apparently somewhat displaced in a downward direction, as though refracted by gaseous matter associated with the filament prominences. Attention is especially attracted to this locality, both on account of its equatorial position and also from the large and complicated prominence forms existing here. Extending above these associated prominence masses is the most extensive series of coronal arches visible at this eclipse.

P. A. $264^{\circ} .5$ The chromosphere in the sector between this position and the Lat. $+7^{\circ} .0$ one last stated is serrated. These serrations have a tendency towards a constantly increasing inclination in an equatorial direction. At the position angle here given a bright slender prominence of about $20^{\prime \prime}$ elevation is seen. This prominence appears to be affiliated with the "fantastic" group already described by an almost evanescent filament. The inclination of the bright prominence at this position angle is such that it is apparently the line of termination of the outer filament extending over the former group.

P. A. $274^{\circ} .0$ From this position angle to P. A. $282^{\circ} .0$ the chromosphere has Lat. $+16^{\circ} .5$ an undulatory or "billowlike" outline. At the given position a small prominence is visible. With this exception the chromosphere is undiversified by eruptive elevations.

P. A. $282^{\circ} .0$ A medium-sized flat-topped prominence is here observed. A Lat. $+24^{\circ} .5$ bright filament of prominence matter extends for a short distance towards the equator from the top of this prominence. This filament curves downward and meets the chromosphere after a short course. 
P. A. $291^{\circ} .5$ This prominence has an elevation of approximately $30^{\prime \prime}$ above to $296^{\circ} .0$ the chromosphere. This is about half the elevation of the promiLat. $+34^{\circ} .0$ nence next to be described, to which it is closely joined at its to $+38^{\circ} .5$ base. The structure of this prominence would indicate that it is subject to violent eruptive forces. These evidences show a repulsion away from the large twin prominences next described. Several filaments of rather bright prominence matter are apparently ejected from this prominence in a nearly horizontal direction towards the equator. The form of these filaments, however, may indicate that they are merely the resulting débris of the general eruption of prominence matter in this region. . In that case the apparent ejection of these filaments is due solely to form and proximity to the large prominence with which they are apparently connected. These filaments extend to the previously described prominence at P. A. $282^{\circ} .0$. The space underlying these filaments is occupied by a long prominence of slight elevation, apparently in contact with the chromosphere at its base. This low prominence exhibits considerable detail of structure.

P. A. $296^{\circ} .0$ to $303^{\circ} .0$

Lat. $+38^{\circ} .5$ to $+45^{\circ} .5$

This sector contains one of the enormous bright prominence groups of this eclipse. It is closely associated with the prominence described above. On Negative 6 it is seen to be built up of numerous individual prominence flames, thus presenting great detail in structure. These associated flames, emanating from a wide base, are apparently interwoven, drawing somewhat closer together as they ascend. The middle part of this aggregation is of a somewhat thinner texture than the rest of this prominence group, indicating a twin or divided structure. This enormous prominence group has an elevation of about $60^{\prime \prime}$, or 27,000 miles, above the chromosphere. A long spray of prominence matter of considerable brightness is seen to be emitted from near the summits of each of the twin parts described. These sprays are slightly arched and nearly parallel. They extend over the prominence previously described, beginning at position angle $291^{\circ} .5$. The lower one of these sprays, that emitted from the part of this double prominence nearest the equator, is the more extensive and much brighter than the other.

The prominence groups extending from P. A. $282^{\circ} .0$ to and including the present sector have a general similarity of appearance both with respect to texture and intensity. The sprays of prominence matter described in connection with them also have a general resemblance. These phenomena show the underlying solar strata to be greatly disturbed in these localities. The corona above this region also shows unusual intensity, together with evidences of a highly perturbed condition.

P. A. $303^{\circ} .0$ The sector between these position angles shows but slight chromoto $314^{\circ} .0$ spheric agitation. A serrated appearance of the chromospheric limb Lat. $+45^{\circ} .5$ is here observed. Numerous emanations of faint coronal matter are
to $+56^{\circ} .5$ is 
seen emerging from the summits of many of these serrations. An inclination of these serrations exists towards the north. As the distance from the equator increases the inclination of these serrations becomes somewhat greater.

P. A. $314^{\circ} .0$ The region included between these two position angles is devoid of to $345^{\circ} .0$ prominences of appreciable size. At P. A. $314^{\circ} .0$ the chromosphere

Lat. $+56^{\circ} .5$ to $+87^{\circ} .5$ begins to be obscured by the Moon on Negative 6, the last of the exposures with the 65 -foot instrument. Serrations similar in type to those observed in the preceding sector are to be seen at intervals above the Moon's limb in this region.

P. A. $345^{\circ} .0$ This is the position of a diminutive group of prominences close to Lat. $+87^{\circ} .5$ the Sun's northern pole. That part of the group nearest the pole is quite small, and shows only a slight condensation of prominence matter existing at a low altitude. The part more distant from the pole is seen as a comparatively short, bright, vertical prominence, with rounded top. It has winglike formations, branching out on either side from its upper extremities. From structural appearances this part of the prominence group is evidently subject to vortex motion. The unusual condition at this eclipse of prominence groups existing in such close proximity to the Sun's pole is again emphasized. The existence of a much larger group of prominences at the southern pole was previously discussed. The influence of these prominences in these positions upon the character of the polar rays is much in evidence, as will be described later in the discussion of the corona.

P. A. $352^{\circ} .5$ to $355^{\circ} .5$

Lat. $+85^{\circ} .0$ to $+82^{\circ} .0$
This region on the eastern side of the Sun's northern pole shows an irregular group of prominences. The first part of this group, nearest the pole, is of rather thin texture, showing much detail of structure. It is somewhat irregular in form, the upper parts being brightest. This object is somewhat inclined toward the adjacent prominence. The other element of this group is in close proximity to that just described. It is much brighter than the former and is pyramidal in outline, with rather steep sides. It has the aspect of a numerous series of flames branching off from a central axis. These flames are arranged with great regularity. Those near the top of this prominence are shorter than those below, thus producing the pyramidal appearance. Several small projecting prongs are observed where the flames composing this prominence project beyond its general outline. As a similitude this object may be said to resemble a "pine tree" with thick clustering branches. From the top of this prominence a faint spray of prominence matter is seen to rise and curve over the prominence adjacent to it.

Between the above group and P. A. $355^{\circ} .5$ no other prominences are visible. 
Changes in the prominences.-Considerable changes took place in several of the prominences during the progress of the eclipse.

A comparison of the appearances of the prominences from different stations, at intervals during a portion of the above period, is available for discussion in this report. Positives on glass from some of the negatives made by the Crocker,Eclipse Expedition of the Lick Observatory, at Goldendale, Washington, were received by the Superintendent of the Naval Observatory, through the courtesy of its director, Dr. W. W. Campbell. Likewise, copies of negatives by the Lowell Observatory Eclipse Expedition at Syracuse, Kansas, were furnished by the kindness of Dr. V. M. SuIPher. A positive was also received from Dr: J. A. Miller of the results obtained by the Sproul Observatory Expedition at Lawrence, Kansas.

At these stations clear sky prevailed and the resulting photographs were remarkably fine and full of detail. The long focus instruments at each of these stations giving details of the inner corona and prominences furnished photographic copies on glass, which were directly comparable with the results obtained at Baker, Oregon, with a similar though somewhat larger instrument. The shorter exposures of the positives furnished as above are taken as a basis of comparison with the results obtained by the Naval Observatory Expedition.

Some of the prominence changes taking place during the interval covered by the absolute difference in time of totality between the above stations were upon a remarkably extensive scale. This was especially the case upon the Sun's western limb, where the prominence group between P. A. $249^{\circ} .0$ and P. A. $264^{\circ} .5$ shows an extensive degree of activity.

Upon the Lick photographs at Goldendale these prominences are evidently in the early stages of eruption. The brighter portion of the inclined section of this group, curving towards the south, is more attenuated at Goldendale than upon the Baker photographs. This is the part of the group which is partially obscured by the filaments of prominence matter to the south of its base. These filament masses on the Lick photographs exhibit considerable volume and intensity. The Naval Observatory photographs show this same part as broken up into filaments, as previously described in the discussion of the prominences of this region. Upon the Lowell Observatory Expedition positives this prominence group is seen to have disintegrated to a considerable extent. The bright inclined part of this group, leaning to the south, shows changes. While of practically the same general form as before, it has a notably thinner texture, as if in the course of dissolution. The series of filament prominences crossing it, already described, have changed but little in the elapsed interval.

On the Lowell plates, the prominence observed at Baker in P. A. $264^{\circ} .5$, has disappeared. This bright, slender prominence on our negatives was associated with the outer one of the bright arching filaments extending over from the "fantastic" group just specified; as that filament has practically vanished in the vicinity of the bright slender prominence, we have further evidence of the changes, or at least of the variable eruptive forces, of this region during this same interval of time.

At P. A. $236^{\circ} .5$, the prominence described on the Naval Observatory plates evidently is in a more active state of eruption than upon those of the Lowell ex$101141^{\circ}-26-$ PT II- -63 
pedition. On these latter positives it is seen as a bright pinnacle, extending nearly straight upwards, and about double its previous height as observed at Baker. Filament prominences are seen on the Lowell photographs, on either side of this pinnacle and rising to a much greater elevation. The coronal features in this region have been compared upon these two photographs which were separated by a considerable interval of time. No evidence of appreciable change either of coronal forms or brightness could be detected in this neighborhood.

A slight change is noted at the summit of the tall spiral prominence associated with the pyramidal group at P. A. $49^{\circ} .5$ to P. A. $55^{\circ} .5$. Upon the Lowell plates the winged tip of this feature. shows considerably more horizontal extension on the northern side than that observed upon the plates of the Naval Observatory.

At either of the polar regions the prominences previously described show but little activity. They may be classed as of the quiescent type of prominences, which are to be expected in these localities. Some slight changes in the appearance of the prominences are noted in the polar regions, upon comparing the photographs taken at these different stations, as might be expected. These variations, however, are upon a very limited scale and relatively unimportant.

In the region extending from P. A. $282^{\circ} .0$ to P. A. $303^{\circ} .0$, but little change is noted upon comparison of the Baker, Oregon, and the Syracuse, Kansas, positives. This fact is especially noteworthy, considering the great size of the prominence groups existing in this sector and the amount of detail exhibited therein. The coronal structure above this latter region, described elsewhere, shows evidence of great solar activity.

The above facts in relation to changes in prominence structure at this eclipse are extremely interesting. Of the total solar eclipses observed by Naval Observatory parties in recent years none have shown variation of the prominences in the time interval available comparable with those here noted.

The inner corona and its relation to the solar spots and prominences.-The inner corona at this eclipse presents many interesting features, together with an unusual amount of detail. The corona is much brighter upon the western than upon the eastern side of the Sun. It is also much broader on the western side. The aggregated western coronal rays extend outward in a wide brushlike formation, while those on the east form a broad tapering wing. The general outline, therefore, of the equatorial streamers is roughly triangular or "heart" shaped. This characteristic was noted when the corona was seen by the unaided eye at the Baker station and confirmed by its appearance on the photographs.

This is the form of corona to be expected during the period of the sun-spot cycle tc which this eclipse belongs. The sun-spot maximum occurred early in 1917. In the frequency of solar spots a considerable decrease was noted in 1918. At the time of this eclipse there were four groups of solar spots visible. These were recorded by the two photographs taken by Mr. G. Harrison with the photoheliograph at the Naval Observatory during my absence with the eclipse expedition. 'These photographs of the Sun were made practically at noon, seventy-fifth meridan standard time, on June 8, or about 6 hours before totality, at Baker, Oregon. 
The Sun was free from spots on its eastern and western sides, near the limb. The spots observed on these photographs, their description, latitudes, and position, in terms of the daily rate of solar rotation, are as follows:

(1) Medium group, near equator, 4 days from west limb.

(2) Small group, south latitude, 4 days from west limb.

(3) Small group, north latitude, 4 days on.

(4) Two large spots, north latitude, 4 and 3 days from west limb.

These solar photographs, taken at the Naval Observatory, show an unusual area of bright faculae in the region of group (4). This faculous matter also extends in a broad irregular zone from those areas surrounding this pair of large sun-spots to the region of the western solar limb. It is noteworthy that the northwest quadrant, overlying the location of this same disturbed faculous belt, shows the greatest amount of agitation and detail in the corona. Other bright faculous masses in the southwest quadrant appear near the solar limb. These are less extensive than those previously described, but from their positions and intensities might account for the enormous "fantastic" prominence groups previously described. Here the inner corona is bright and extensive. It shows in this locality a remarkable amount of detail not apparent in the northwest quadrant.

These solar photographs made at the Naval Observatory have been carefully examined for faculae in the vicinity of the north and south poles. This search, however, was without success. Faculae are seldom observed in these polar regions of the Sun, although the poles are visible alternately at semiannual periods. If detectable they would have had an important bearing upon the prominences observed at either pole. Photographs made on a large scale with the spectroheliograph at about the time of the eclipse where the Sun was unobscured might give some data on the subject. If unusual conditions in these polar regions existed at the time, such methods of observations should detect them. On the eastern side of the Sun an area of bright faculous matter is seen near the solar limb near to but north of the equator. This region corresponds approximately to the center of emanation of the broad tapering wing, the principal general feature of this locality in the middle corona.

No photograph was taken of the Sun on the day succeeding the eclipse at the Naval Observatory. The photograph of June 10, however, shows an increase of faculae in the region which would have been situated near the eastern limb of the Sun on June 8, the day of the eclipse. It also shows that with the exception of a group of bright faculae in the southeast quadrant in the sun-spot zone that the region near the eastern limb is less marked by faculous matter than upon the day of the eclipse. The area occupied by the bright faculae above referred to would have been located near the solar limb if existing at eclipse time. But one bright and conspicuous prominence was observed in this northeast sector at this eclipse. There is also a large development of bright coronal matter in this locality which evidently had some dependency upon the faculous disturbance in this neighborhood. These faculous areas, in general, are known to be quite constant. While the individual faculous markings are seen upon the photoheliographic records to have changed in form to some extent from day to day, the areas of disturbance themselves usually remain practically the same for considerable periods of time. Solar spots and 
groups are mostly accompanied by faculous areas of greater or less extent. As faculae have also a much wider distribution in latitude than the spots, it is reasonable to expect that the former will have a predominating influence upon the corona, its distribution and disturbances, rather than the spots themselves. The intimate connection apparently existing between the faculae near the limb of the Sun and the detailed features of the corona at this eclipse would seem to emphasize this fact.

The irregularities of slight elevation observed upon the limb of the exposed chromosphere upon photographs taken near the beginning and ending of totality are undoubtedly of the same general character as the "billows" of the polar regions. These "billow" formations have been described and discussed in connection with coronal observations by Naval Observatory parties at the eclipses of 1901 and 1905 . At the present eclipse, as previously stated, the location of the station close to the central line of totality prevented observations of this character at the poles of the Sun, such as were made upon those former expeditions. Upon Negative 1, however, the southeast sector of the projecting chromosphere is seen. Likewise, on Negative 6 the northwest sector of the chromosphere is exposed to view over a considerable arc. Upon these negatives, as previously stated, a "sierra" formation is noted at many localities on the chromospheric limb. A detailed examination relative to the corona shows that these sharp-pointed elevations, which become attenuated and diffuse at their upper extremities, are obviously composed of coronal matter. The coronal matter thus emitted is bright, highly condensed, and of relatively slight elevation on these negatives with short exposures. It is likely that the thin cloud-haze prevailing at our station at this eclipse, acting as an absorbing screen, somewhat accentuated the contrast and visibility of these inner features under the conditions described.

In many instances, on the photographs; beneath these sharp-pointed objects, irregularities of a distinctive character in the chromosphere itself are observed. These elevations, while somewhat irregular, both in regard to size and distribution, have a general similarity of form. Moreover, as the polar regions are approached, these slight chromospheric elevations take on an appearance more like the "billows" heretofore observed near the poles of solar rotation on the previous expeditions referred to.

In several regions where low-lying prominences of considerable lateral extent occur, especially near the equatorial regions, these "billows," or the serrated form of chromospheric limb, are absent. These low-lying prominences without eruptive features are generally of the quiescent type, often remaining visible by the usual spectroscopic methods without change for considerable periods of time. The region to which this remark especially applies is located just south of the "fantastic" prominence near the equator, on the west side of the Sun. This sector in part, as previously remarked, is the locality of an intense coronal disturbance. This disturbance in the corona, however, does not conform in aspect with these low-lying prominences. It probably has for its origin, therefore, a position on the solar surface either in front of or behind them, and consequently does not originate at the Sun's limb. 
Moreover, none of the typical emissive jets emerging from these low-lying prominences are noticeable in this locality, indicating that the region adjacent to the solar limb is in a comparatively quiescent condition.

It is likely that our knowledge of solar surroundings exterior to the photosphere will be principally derived from the study of the inner coronal regions at each succeeding eclipse. The Sun is the only star to which we have such direct access. Here among the multitude of stellar bodies we are able with suitable apparatus to investigate surface conditions, and surroundings. The occasions for obtaining this observational material for study of the solar coronal regions by present methods are only to be had at comparatively infrequent intervals, and often by transporting apparatus and personnel to distant parts of the Earth's surface within the Moon's shadow.

The Sun has been studied visually and photographically for many decades with respect to its markings. Its chemical, physical, and associated conditions are much better understood now than formerly. The prominences are now observable spectroscopically, or are made to record themselves by the improved spectroheliographic methods as easily as the spots or faculae were formerly observed. The speed of solar rotation is known for different latitudes by several methods of determination, and many theories with regard to this luminary not here specified are accepted as facts.

Much at present is known either hypothetically, or as an accepted fact, with respect to the origin and conditions existing in the coronal envelope. The coordination of observed conditions and phenomena existing at each succeeding eclipse by improved methods of the future will more clearly elucidate these conditions. If finally some method is devised for successfully recording the appearance of the corona without an eclipse, our knowledge of the conditions obtaining in these solar surroundings can be much improved by continuous observation. However, on the rare occasion when total solar eclipses are observed, the changes of aspect and varieties of form and details, together with the general type then observed, render it possible to more easily trace to their source the causes of these variations.

So far as the writer is aware no changes in the features of the corona have been detected with certainty at any one eclipse. This has been attempted on several occasions by comparing photographs made at widely separated stations involving a considerable lapse of time between the observations. The important and even rapid changes in the size and shapes of some of the prominences under these circumstances are in marked contrast to the apparent slow variation in the coronal features in their vicinity. This lack of success in identifying coronal changes under these circumstances, even when large and powerful instruments are employed, may be due in part to the more ephemeral and indefinite markings of these coronal features.

There is no question, however, but that changes of coronal form and detail take place at a much less angular rate than those of the chromosphere and prominences. It is practically certain also that no extremely rapid changes of brightness take place during the period available at different and widely separated eclipse stations. 
Owing to the intense forces to which these inner coronal regions are subjected, an analysis of the conditions observed in these localities becomes extremely complex.

A brief summary of the forces acting in the coronal and subcoronal regions may be of interest in connection with this discussion. The following facts have been recognized as being of special importance in relation to the forces and conditions governing directly or indirectly the distribution and circulation of matter in the corona.

We may first consider the force of gravitation at the Sun's surface about 27.6. Opposed to this gravitation are the projectile forces emanating from and originating principally below the chromospheric layer. These are owing to the difference of mechanical potential due to solar radiation and the molecular efforts of the gaseous substratum to a considerable depth to reestablish equilibrium. This condition, as is well known, is the basic one for most of the phenomena observed upon the visible solar surface and its surroundings. It is primarily accountable for existing conditions. As the subsurface gases arise and mingle to maintain this radiating and thermal equilibrium they expand as pressure of the superposed layers is relieved, though still subjected to enormous compression. This expansion, together with the currents engendered in the viscous mass, produce areas of convection currents of greater or less violence in the subvisible solar surface which give rise to many of the observed phenomena in the photosphere. The nearly constant energy loss from these upper areas produces the light, heat, and other wave-lengths of energy radiated into space. Overlying the photosphere we have first the reversing layer, the superposed chromosphere, and the overlying prominence elevations. These latter objects are the visible effects of the violent commingling of the ultraheated matter of the photospheric regions which have communicated their mechanical energy to the superposed parts. In other terms, explosions are taking place, or regions of abnormally high outward pressure exist, in the localities where the prominences are formed upon a greater or lesser, but always stupendous, scale. The gaseous matter and metallic vapors composing these prominences, in their violent ascent, carry with them vapors of a lower temperature than themselves and the lighter gases of the corona. This fact is proven by the coronal arches which are found spanning these large eruptive prominences. It is likely that these arches are really "dome" shaped when considered in terms of three dimensional space. They may be explained as the result of successive explosive impacts which distribute the matter in this common arch or domelike form.

But other factors are recognized in these regions, such as light pressure, magnetic conditions, as well as the purely mechanical effects previously considered. Just what the results of these last forces would produce but little is at present definitely ascertained. The polarization of light from some parts of the corona has been observed. This fact would indicate that solid matter exists in or composes parts of this nebulous mass. This solid variety of coronal matter is probably in a finely divided state. The pulverized condition probably existing may result from the abrasion of the particles of uprushing matter colliding with those which are falling back to the solar surface. This condition, together with the rapid diminution of the previously mentioned opposing forces and lack of material as 
the distance from the chromosphere increases, would account for the slow change of coronal forms. As the metallic vapors are projected into space by the explosive forces existing, liquefication, or even solid matter, might result from the released pressure from elevation. This metamorphosis would also be favored by a recession from close proximity to the chromosphere, where the observational evidence shows that partial condensation is taking place, compared with the more intensely heated layers below. These remarks apply principally to the inner corona, of which a detailed study at each succeeding eclipse is extremely desirable for purposes of comparison of relative coriditions existing.

The outer corona and its extensions are probably merely extreme manifestations of the forces evidenced in the interior portions. This is predicated from the fact that the streamers of the outer corona are fainter extensions of those observed as more intense wings, or rays, in the inner corona. These inner features may act individually or in combination to produce the streamer effect. The brush form of streamers observed at this and previous eclipses seems to indicate that the emanating forces are distributed over a wide range of latitude, both north and south of the spot-zone regions, near the limb, as existing at the time of the eclipse.

The foregoing remarks apply principally to the equatorial zones. At the poles a different condition exists which has been described by the writer in the report on the eclipse of 1901, as observed at Fort de Kock, Sumatra. This was published in a preceding volume of the Naval Observatory publications. Briefly, in the polar regions the coronal rays are seen to emerge in many cases from the tops of slight wavy elevations of the chromosphere. These elevations were at that time distinguished as "billows" by the writer, for want of a better term. The observations of objects on the projecting chromospheric limb have been previously described for this eclipse. Billow-like formations are seen near the poles upon these negatives made at the Baker Station, as on former occasions. A serrated limb in the spot zone and adjacent regions was also observed upon these negatives. In each of these cases bright jets of coronal matter were visible issuing from the summits of these elevations. These billows, and likewise the serrations, are then the probable source of much of the matter composing the corona, especially the former or billowlike elevations of the chromospheric limb in the vicinity of the poles.

Regarding the absolute temperature of the Sun there has been considerable discordance in the results arrived at by different methods and observers. We must bear in mind, however, that these observations depend upon the radiation constant of the visible surface and surroundings. The temperature undoubtedly is variable to some extent, either in part or as a whole. What the absolute temperature may be at even comparatively slight depths below this observable surface, we have no adequate conception except from theory.

At these lower depths it is agreed that the temperature would be materially increased. The ratio of the increment would depend upon the differential of the radiating capacity of the matter composing these lower strata.

While undoubtedly the body of the Sun below the photosphere is a true gas at the temperatures existing in that body, owing to the compression of the molecules 
composing it there is doubt if the laws of gases in this case are strictly followed as the Sun's center is approached

Owing to the plastic condition of these subsurface gases, convectional currents would be impeded, and radiation would follow more nearly the laws of radiation in the case of solid masses. That implies that the temperature of the Sun's central core may be transcendental, compared with any recognized degrees of heat in those parts accessible to observation. According to LANE's Law a purely gaseous body like the Sun by contraction upon cooling by radiation becomes hotter. In this paradoxical condition, as the diameter of the body becomes smaller the inner parts near the center would first lose their purely gaseous character by compression and become of a plastic nature. This critical period would ensue when the increase of temperature according to LANE's Law would be insufficient to overcome the compression due to the gravitational pressure of the superposed mass of the body. Therefore, in the case of the Sun as it exists at the present time supertemperatures are likely to be found at a comparatively slight depth below the visible photosphere. This would account in part for the coronal jets observed emerging from the billows or serrated edges of the chromosphere, as seen in relief at its limb. It would likewise account for the long duration of solar existence with a radiation nearly constant. If these conclusions are true, geoglogical and astronomic periods of time required for observed evolution are rendered more in accord. At the present epoch, more space and more time, with much greater span is required to coordinate the different branches of science with the observed works of creation. The assumption for the Sun of a much more highly heated interior than formerly recognized will serve to extend the span of its life history considerably. It will also defer the period when, by radiation, the Sun will no longer be able to support life as at present existing upon the Earth, and tend to harmonize our newer concepstions of the universe.

\section{SPECTROSCOPIC WORK AT THE 1918 ECLIPSE.}

By Dr. S. A. Mitcheld.

The excellent photographs of the flash spectrum obtained in 1905 by the use of the concave grating pointed the method to be followed in 1918. Accordingly, attempts were made to photograph the flash spectrum with three concave gratings of widely different dispersion, as follows:

A 21-foot concave grating, to be used in the first order; a 10-foot concave grating, to be used in first order; a 1-meter concave grating, to be used in first and second orders.

The purpose of the work in 1918 was to duplicate the results of 1905 and to extend the region of investigation for the ultra-violet as far into the red as possible. The grating used with success in 1905 was of 4 -inch aperture and 10-foot radius of curvature.

Through the kindness of Prof. J. S. Ames of the Johns Hopkins University a 6-inch, 21-foot concave grating, unusually bright in the first order, was loaned for the purpose. It was hoped that the exposure time would be sufficient to be able to use the increased dispersion. If successful photographs had been obtained with good 
dispersion they would have been used in a detailed study of the spectrum lines in order to determine the wave-lengths with greater precision, and to measure the heights to which the various vapors ascend. Dr. C. E. K. Mees of the Eastman Kodak Co. kindly coated heavy celluloid films with a specially prepared emulsion, $\mathrm{E}-4$, in order to obtain as great an extension into the red as possible.

The films used with the 21-foot grating were, in size, 2 by 24 inches, and an attempt was made to photograph from $\lambda 3300$ in the violet to about $\lambda 6500$ in the red. Four films were arranged in a plate-holder parallel to each other, and two plateholders were used, so that provision was made for eight exposures. With considerable practice the plate-holder could be shifted rapidly from one film to the next without shaking the box. This box, shaped like a grand piano, was made of mahogany, well seasoned. Light from the coelostat mirror entered the box, then fell on the grating, and after reflection was brought to a focus on the photographic film. In order to secure a normal spectrum the photographic film was placed at right angles to the radius of curvature of the grating. Where the light entered the box a flap shutter was made that could be quickly opened and shut by hand. Exposures were made in this manner by Dr. L. G. Hoxton, professor of physics at the University of Virginia. Miss Harriet W. Bigelow, Director of the Smith College Observatory, shifted the plate-holder.

The 10-foot grating used in 1918 differed from the one of similar radius used in 1905 by being of 6 -inch aperture instead of 4 . The grating belongs to the U. S. Naval Observatory. The films used were similar to those with the 21-foot grating, the special emulsion, E-4, and the size of the films $1 \frac{1}{4}$ by 14 inches. The length of the film was sufficient to permit photographs from about $\lambda 3500$ in the violet to $\lambda 7200$ in the red. It will be seen that the 10 -foot grating was arranged to photograph further into the red end of the spectrum than the 21-foot. Six films were arranged in each of two plate-holders so that twelve exposures were available. On eclipse day the exposures were made by the writer, who was ably assisted by Miss Mary MurRay Hopkins, associate professor of astronomy at Smith College, who shifted the plate-holders between exposures.

The third concave grating was of very small dispersion from a radius of curvature of one meter. The grating was of 6 -inch aperture kindly loaned by Dr. C. G. Аввот, Director of the Astrophysical Observatory of the Smithsonian Institution, and the spectra from it were very brilliant. Dr. PAUL W. Merrill was in charge of this instrument, and through the kindness of Dr. S. W. Stratron, Director of the Bureau of Standards, he was provided with a supply of dicyanin to stain the films coated with SEEd 30 emulsion. An attempt was made to photograph to wavelength $\lambda 9000$ in the red. Color filters were obtained from Doctor Mees to absorb the overlapping spectra of second and third orders, these filters being on thin gelatine. G. J. Pehling, MM2C, N. R. F., assisted Doctor Merrill on eclipse day by shifting the plate-holders.

The 21-foot grating and the one of small dispersion were housed in the same tent, the light to the two instruments being fed by the two mirrors of the Gaertner coelostat, the large grating using the upper mirror. The grating of 10 feet was under a separate tent, and one of the coelostats of the transit-of-Venus was employed. 
The three grating boxes, the plate-holders used, and all accessories were sent to the University of Virginia early in the year, and all necessary alterations to the apparatus were made in the shop of the department of physics with the able assistance of Dr. L. G. Hoxton.

At Baker the instruments were set up by Doctor Hoxтon and the writer, assisted by the sailors of the United States Navy. The three spectrographs were focused by the same collimator used with success in Spain in 1905. This collimator consisted essentially of a slit arranged between two parabolic mirrors. On the day of the eclipse all of the three gratings were used as objective instruments, or in other words, without slit. The excellent focus obtained in 1905 left nothing better to be desired. The writer, assisted by Doctor Hoxton, attended to the focus of the 10-foot and the 21-foot grating, while Doctor MerRILl and Doctor Hoxton focused the grating of small dispersion. Difficulty was experienced with the focus of the small instrument on account of the astigmatism from the short focus.

In order to tell the time of beginning of totality the writer used with success in 1905 a pair of binoculars. Before one of the objectives a direct-vision spectroscope was arranged consisting of a plane grating and plane mirror, while the other objective was left free. Armed with this it was possible to see with the left eye through the free objective the disappearing crescent of the Sun as totality approached. With the right eye and the direct-vision spectroscope it was possible to see the bright.lines of the spectrum as they gradually disappeared. At the eclipse of 1905 with clear skies the bright lines were seen to appear gradually, and the signal for the beginning of the program starting with totality was given from the appearance of the flash spectrum.

In 1918 on account of the thin clouds it was impossible to see any of the bright lines, and the signal for the beginning of totality. was actually given by Mr. HAMmond from the 5-inch telescope.

The developed films were a great disappointment. The clouds had tremendously cut down the light. On the films of the large grating nothing whatever was visible. With the 10-foot grating on the film of the second flash quite a number of the strongest lines were visible, all in sharp focus. Some lines appeared with the grating of short focus, but none of the interesting lines in the red with which we were specially interested.

The spectra of 1918 have not been measured, since the spectra were weak and so inferior to those obtained in 1905. Unfortunately for the progress of eclipse spectroscopy similar conditions met the other observers who were employing instruments of high dispersion. It will be necessary to wait for the next eclipse to attempt to carry out a similar program.

\section{PAINTING THE SOLAR CORONA.*}

\section{By Howard Russell Butler, N. A.}

Illustrations from drawings which give the artist's records made at the time of the eslipse and explain his method of work: also from the artist's paintings of the phenomena of the eclipse, color plate opposite, and frontispiece in color "Total Solar Eclipse of June 8, 1918."

In May, 1918, I received an invitation from Mr. Edward D. Adams, well known as a patron of science and art, to accompany him to Baker, Oregon, where the United States Naval Observatory had established its station for observing the

* Reprinted from Natural History, Vol. XIX, No. 3, pp. 244-271, 1919. 
APPENDIX, VOL. $X$, PUBLICATIONS OF THE UNITED STATES NAVAL OBSERVATORY.

PLATES XVI-XVII.

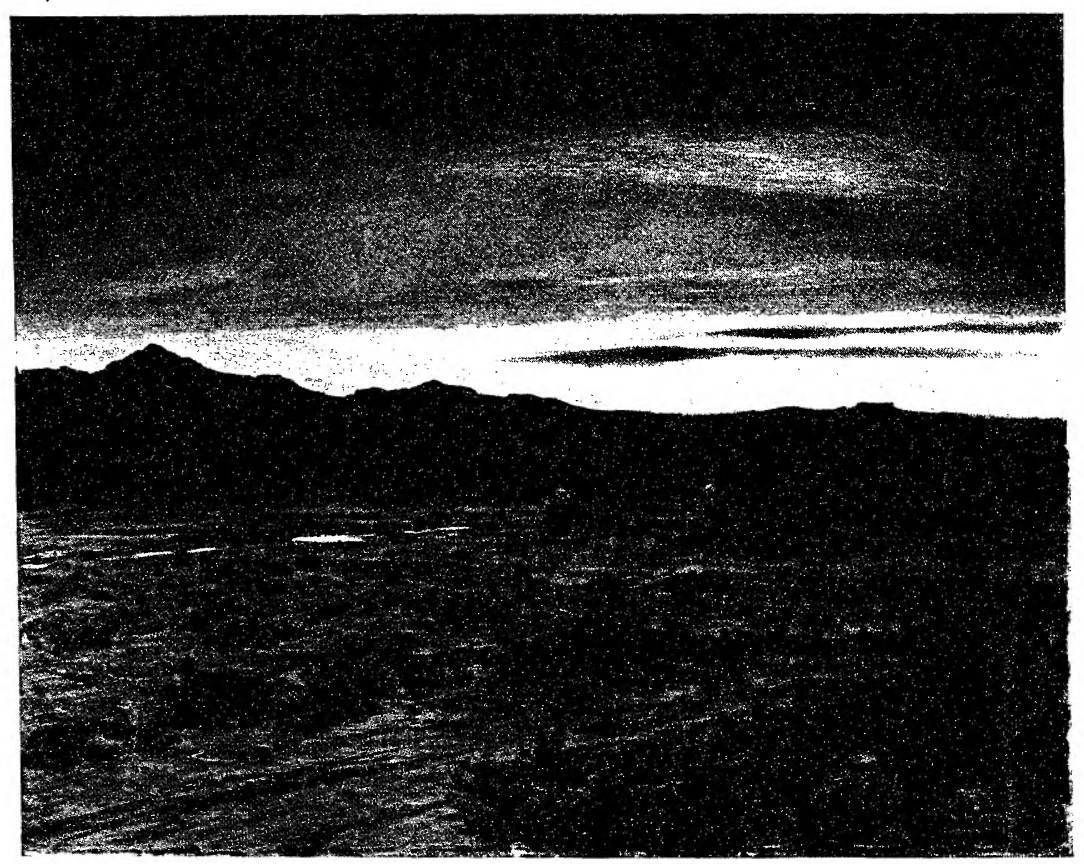

THE APPROACHING SHADOW OF THE MOON, BAKER, OREGON, JUNE 8, 1918.

From the Painting by Howard Russell Butler, N.A.

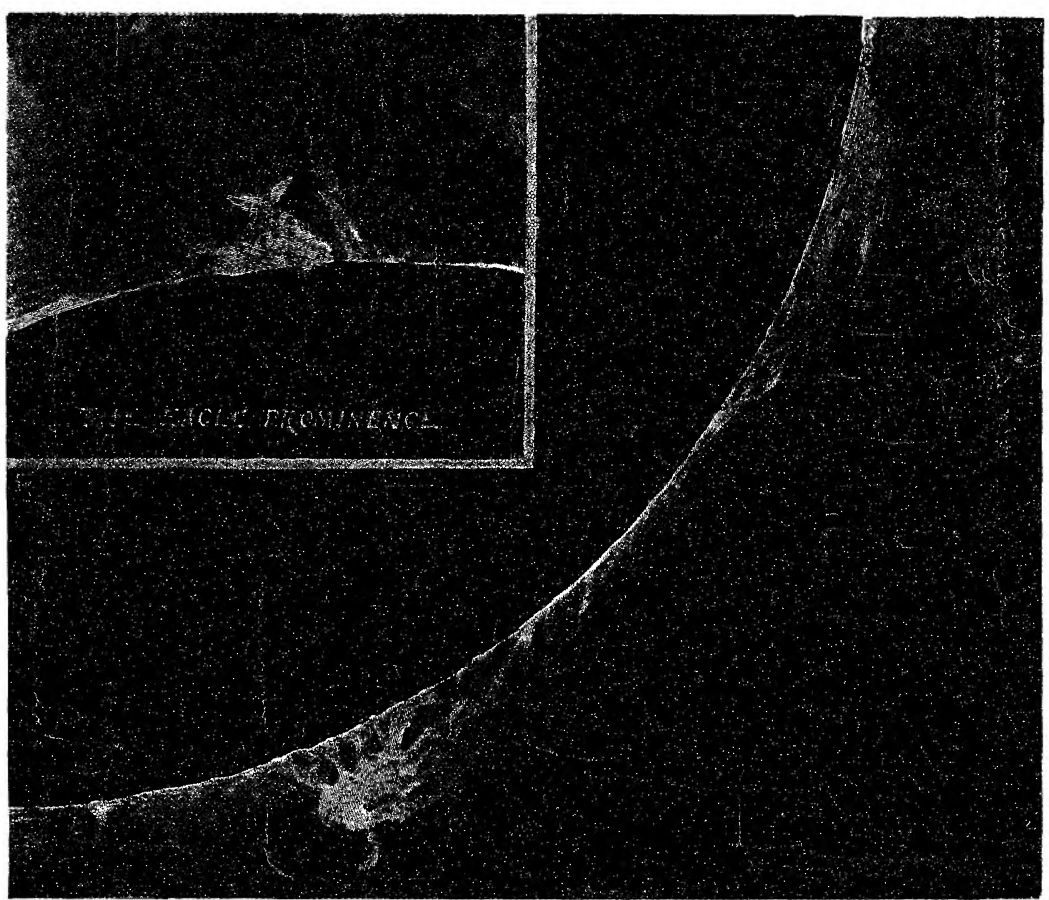

DETAILS OF THE HYDROGEN PROMINENCES, JUNE 8, 1918, INCLUDING THE "EAGLE PROMINENCE".

From the Painting by Howard Russell Butler, N.A. 
total solar eclipse of June 8, 1918. Dr. S. A. Mitchell, Director of the Leander McCormick Observatory of the University of Virginia, and Mr. Adams had agreed that a painting of the corona might be made which would have both scientific and artistic interest.

Many drawings and countless photographs (some colored by hand) have been made of solar coronas, but I was told that no record existed of any painting actually made from direct observation. The invitation was therefore accepted as a unique opportunity.

As a portrait painter I have usually asked for ten or twelve sittings of two hours each; now I was asked to render my subject in 112 seconds. The method of procedure therefore became all important.

The first step was to study the reports in astronomical and popular works of previous eclipses and thus familiarize myself with all attempts to describe or record the form and color of the corona and prominences. Of these attempts there are a great number. They describe an outer corona, varying in extension from a fraction of a diameter of the Moon to many diameters, the color usually being described as pearly and variously tinged; an inner corona, more brilliant than the former; and the prominences of incandescent hydrogen, variously described as red, ruby colored, pink, and blood-red. In addition to these, my picture would have to show the dark surface of the Moon, and the sky with whatever color value it chanced to have at the moment of observation.

As regards the shape and the extension of the outer corona, a theory exists that it varies inversely in size as the combined area of sun-spots, and this seemed to be confirmed by about twenty drawings of previous eclipses, which I made from photographs and prints and reduced to the same scale. Thus in the eclipse of 1900, when sun-spots were at a minimum, the corona exhibited wide extensions, having interesting shapes, two of which became known as the "Angel Wing" and the "Herring Tail". extensions. As the number and size of sun-spots seem to vary quite regularly, so that the maximum is reached about every eleven years, and as we were near a maximum period, wide extensions of the outer corona were not to be looked for. We expected about three-fourths of a diameter on each side and this is about what we saw.

All reports of the so-called "inner corona" agree that the part nearest to the Sun is very brilliant and this inner corona is usually described as whitish in color. The transition from this inner portion to the far less brilliant outer part is quite abrupt, but one of the questions on which there seems to be a difference of opinion concerns an absolute demarcation between the inner and the outer coronas. I found none.

As regards the prominences-while often discernible with the naked eye, it is necessary to have a good glass to get the details of shape and to study the color rightly. The Naval Observatory put at my disposal a fine pair of ZEIss binoculars, which proved of the greatest value. I realized in advance that my hardest task would be to portray these prominences in their proper color and brilliancy. According to Professor Mitchell, I was to expect them to have a color not unlike that of the hydrogen line $\mathrm{H}_{\alpha}$ in the spectrum, possibly slightly modified by the much fainter 
bluish line; and ample opportunity to study these lines in the spectroscope was given to me. How best to render this color in paint and to give it its luminous character was the problem. Realizing that this would necessarily be the brightest tone in the picture and that it would have to stand out brilliantly against the tone of the inner corona, also bright, I set to work to produce the brightest possible red; that is, the one which stood highest in a scale of values of which varnished ivory black was zero and the best lead white (commercially known as silver white) was 100. I tried French pastels and water colors, the latter over Whatman paper, but ultimately found that I could do best with oil paint.

The process of obtaining this red decided upon for the final picture, but which takes more time than I had at Baker, was to prepare a hard surface of silver white, well dried, to paint over that a thin coating of zinc white tinged with orange cadmium, and, when that was perfectly dry, to glaze it richly with rose madder or garance rose doré. This gave the tone with its fiery quality, but alas, its value, while the highest that I could get, was down to from 65 to 70 in the black and white scale. The highest value obtained by mixing wet colors at Baker was about 60 .

Granting this to be the highest note that I could have in my picture, I next addressed myself to the lowest. Would this be the sky or the dark surface of the Moon? Regarding the color and value of the clear sky during solar eclipses there were varying opinions. Many drawings show the Moon as black against a sky represented by a medium gray. These I believed to be incorrect and found them so. The Moon, having a less luminous quality than the sky and surrounded by the brilliancy of the corona, should appear slightly darker by optical illusion. The sky value was at any rate the safer note to work from and, except for the slight variation alluded to in the Moon, it would surely be the darkest value in the picture.

Assuming then a sky value of say 25 and a prominence red value of say 60 , the total variation in values would thus be limited to 35 points-surely a small range with which to reproduce so brilliant a phenomenon.

The method of working finally adopted may be called a shorthand method. It was to have a sheet of white cardboard on the easel with a series of concentric circles and radii drawn upon it in advance. One of these circles was to have the same diameter as the photographs of the Moon to be taken in the 65-foot camera, namely, $7 \frac{3}{8}$ inches. There was to be an inner circle of half this diameter and outer circles whose diameters were respectively $1 \frac{1}{2}, 2$, and $2 \frac{1}{2}$ times that of the inner circle. I expected to use the $7 \frac{3}{8}$-inch diameter, and did actually use it, but I was thus prepared, in case of an unexpectedly extended corona, to reduce the scale to one-half and get everything on the cardboard. In front and beneath my cardboard was a finished sample picture of a corona, painted in advance as I expected it would appear, and my plan was to indicate by initials at points on my cardboard the variations of color from this picture; thus $b$ was to mean a variation toward blue from the sample picture, and $y$ more toward yellow. I wrote out the procedure as follows and tacked it alongside the easel. Practice enabled me to allot a certain number of seconds to each item. 


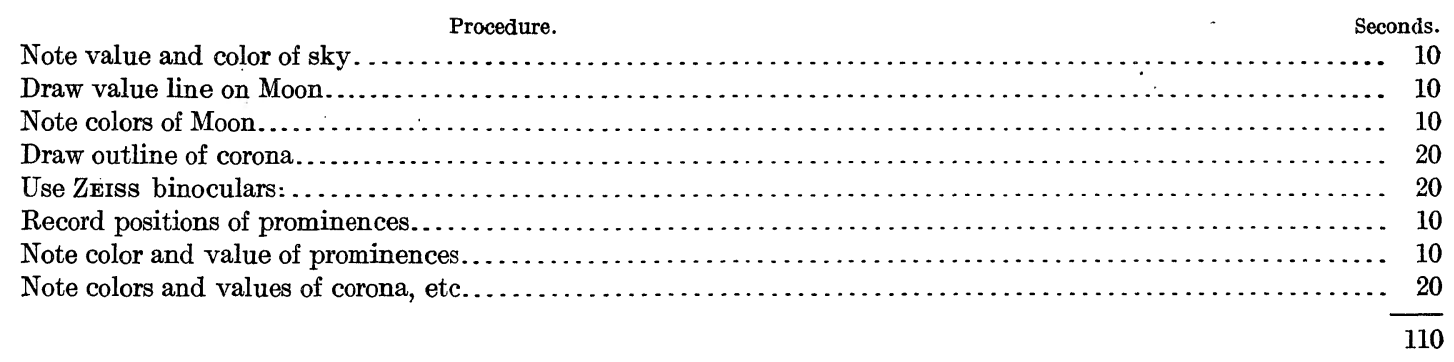

Then my plan was to paint a first picture from this resulting memorandum, while the impression was vivid, and as soon as there was sufficient light to proceed by.

Several methods of still further shortening the process naturally presented themselves, which can best be understood by a simple diagram. Thus let the vertical axis, Figure 1, represent values in the black and white scale and the horizontal axis distances in terms of lunar radii. Then a simple stroke $A$ at 65 gives the value of the prominence, and the added expression $r y$ means "rose very strong with a tinge of yellow." The line $B$ represents the values of the corona. Any distinction between an inner and outer corona could be noted by a quick fall, as at $b$, in the line. Two tangents were drawn in advance on my cardboard for use as vertical axes. The line $C$ would show the Moon to be light in the center and dark on the edges, in this case tinged with green and brown. After the actual experience I cannot think of a better plan than this one.

The observation station was in the fairgrounds at Baker, about a mile and a half from the center of the town. It was surrounded by a wall and low buildings, which insured privacy. A grand stand ran north and south with a double door opening from the top aisle. This door, intended as an exit or fire escape, opened upon a platform with flights of steps descending both ways. This platform was assigned to me, and on it I erected a strong easel and shelves extending to right and left and making an angle with each other. Wind guards and braces were added. The platform faced west and, as the Sun at the time of the eclipse was to be about $12^{\circ}$. south of west, the position could not have been better. It had also a great advantage in being so high up that I could look over the surrounding walls and low buildings and get a fine view of the valley and of the Elkhorn Range in the direction of northwest along the line of the approaching shadow. By keeping the north half of the door into the grand stand closed and boring a small hole through the door, an excellent camera obscura was obtained, the image of the Sun appearing on a tilted white-covered board on the inner side of the door. I had been advised and had determined not to look at the Sun for a considerable time before totality so as to avoid what is known as retina-fatigue, which is certain to result from looking at the brilliant crescent. The camera obscura gave all the information wanted as to the diminishing crescent and yet left me free to watch for the approaching shadow.

As the day drew near drills were instituted, eight or ten of which I attended; each time I went through the procedure as outlined, drawing an imaginary eclipse. The counter, a naval officer, called each minute from 5 before to 1 before, and then gave the call, "30 seconds." The word "Go" was given by Mr. J. C. Hammond, 
astronomer of the Naval Observatory. On the occasion itself this word was given, of course, from actual observation of the eclipse. The counter then called seconds from 1 to 112, when the performance would supposedly be over. These drills were invaluable.

Figure 1.-Total Solar Eclipse June 8, 1918, Baker, Oregon. Short-hand Method of Recording Values.

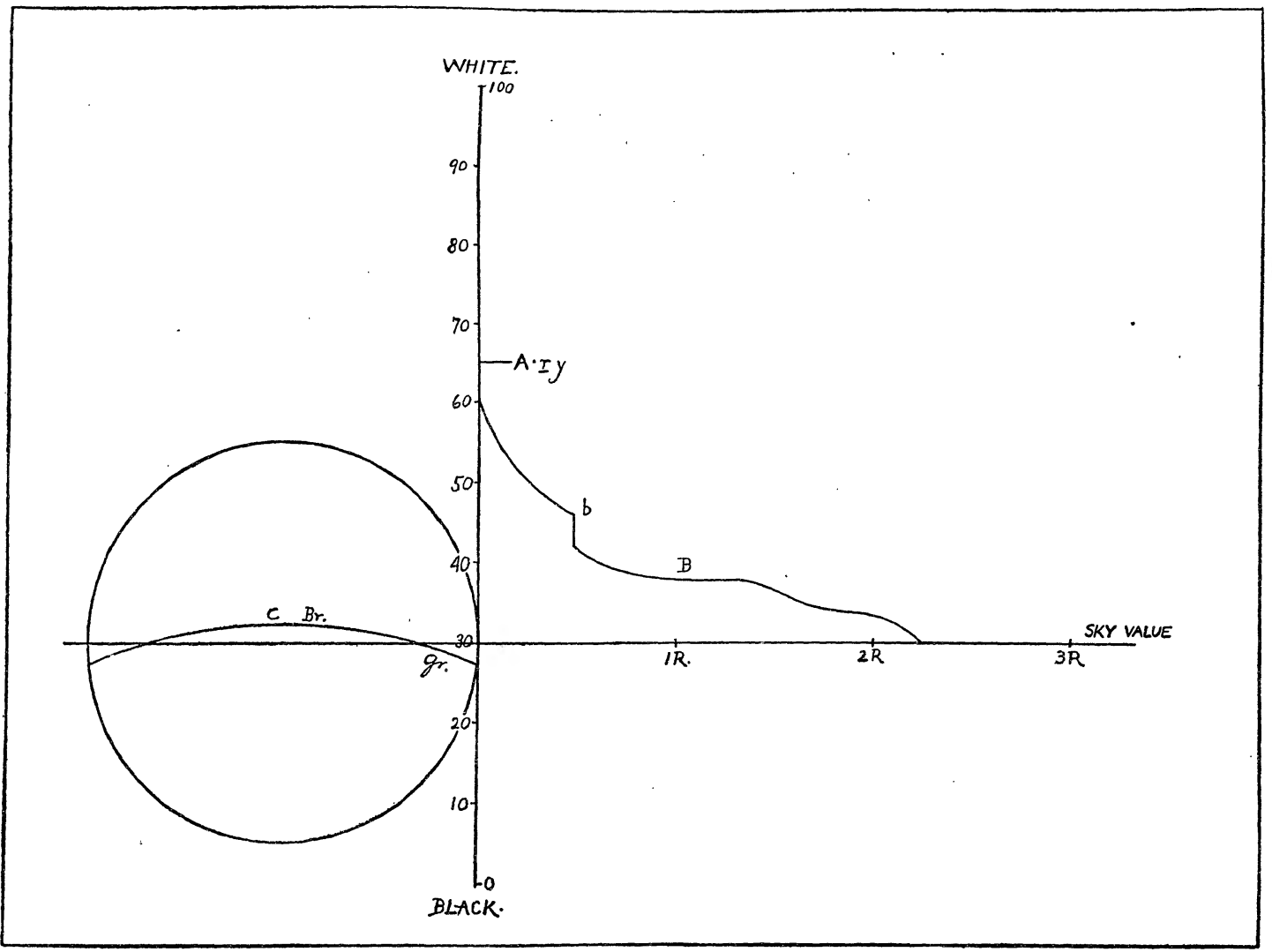

Two diagrams combined, oneillustrating the artist's shorthand method of recording during the few seconds of the eclipse the brightness of the colors in the corona, the other a method of noting the depth of shadow on different areas of the Moon's disk. One diagram consists of the two perpendicular lines, or axes, and the curve B. The vertical axis represents a scale for measuring the brightness of a color, considering ivory black as zero and silver white as 100 . Distance on the horizontal axis measures distance on the sky beyond the Moon's edge measured in radii of the Moon (" $1 R$ " equals a distance of one radius or half the Moon's diameter from the Moon's edge). This horizontal axis is drawn through 30 on the brightness scale, that being the estimated brightness value of the sky during the eclipse. A curve drawn between the axes shows the variation in brightness of the corona at any given point, beginning at the inner edge of the corona and passing outward to the dark sky; that is, the color in the inner corona close to the Moon is 60 on the scale (or in other words the tone of the inner corona is about three-fifths as bright as silver white). From the curve drawn downward from 60 we see that the corona at $1 \mathrm{R}$ (one radius distant from the Moon's edge) had fallen to a brightness of about 40 , and slightly beyond the length of $2 \mathrm{R}$ it disappears, blending with the sky. The line $\mathrm{A}$ is the artist's shorthand toindicate that the prominences had a brightness value of 65 and " $r y$ " is a quick way of recording it if they were "very rosy, tinged with yellow." These two axes were drawn on the cardboards on which the eclipse was to be drawn, in advance, on two sides of the circle of the Moon (see Fig. 2), being represented as tangents to the Moon's circle (see right hand and upper left hand of figure). By means of the curves drawn in on these axes with great speed during the eclipse, we can read off the brightness of the corona's colors at any distance from the Moon.

The line $\mathrm{C}$ in the other diagram (the Moon's disk at the left) is a shorthand way of indicating that the Moon was lighter in the center than at the edges, and that these edges were darker than the sky. The "Br." and "Gr." indicate a tinge of brown and green, respectively.

At first contact, June 8, $2: 47$ p. m., all was ready, but the sky was so cloudy that few of the 18 members of the party expected any good results. A gloom more dense than the cloud overhung the spirits of the camp. But at 3:30 the clouds had grown decidedly thinner, and at 3:50 a large area of blue sky appeared to the right of the Sun. Then the sky cleared so rapidly that all hopes were revived, in the belief that when totality would take place at $4^{\mathrm{h}} 3^{\mathrm{m}} 52^{\mathrm{s}}$ the Sun would be found in an absolutely clear sky. 
Standing with the Sun back over my left shoulder-it was at an elevation of about $45^{\circ}$-I looked at the diminishing crescent on the face of the camera obscura until the call " 1 minute" was heard. Then, turning my eyes to the northwest, I gazed at the north end of the Elkhorn Range and the intervening valley. Roosters were crowing loudly on the neighboring farm; a greenish pallor overspread the landscape-but it was not very dark. To the northwest, however, the sky was growing

Frgure 2.-Total Solar Eclipse June 8, 1918. Baker, Oregon. Original Drawing.

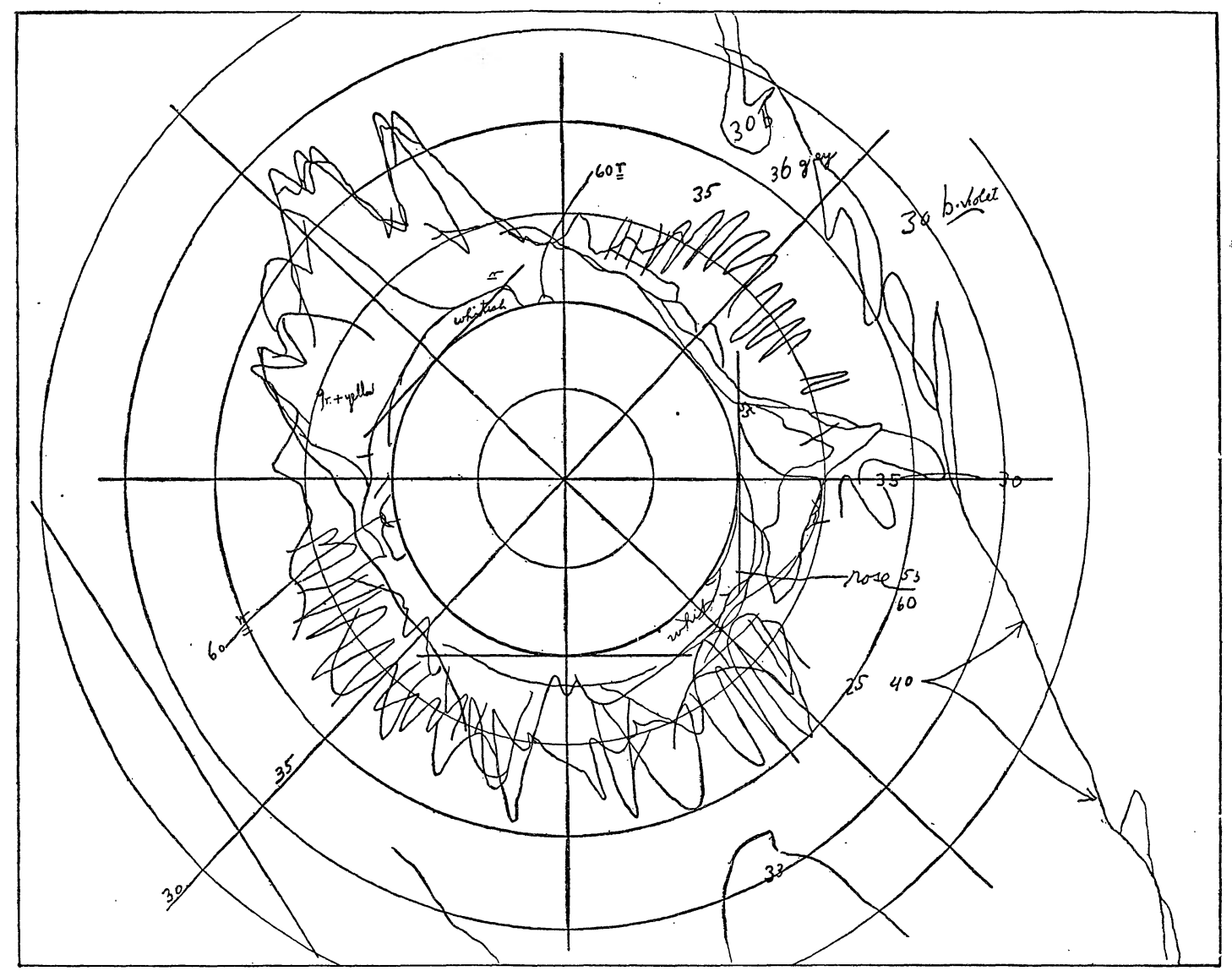

This is a reproduction of the actual original sketch, made at the time of the eclipse, on a cardboard on which the radial lines, circles, and tangeats had been prepared in advance. This is the artist's record, not only of the general outlines of clouds and corona, but also of the colors which are indicated by initials or words (underscored when the colorisintense), and of the brightness of the various parts of the corona, indicated by numbers and by curves such as are explained in the preceding figure. The artist had painted previously a picture of the way in which he "expected" the eclipse to appear and no time was wasted putting in colors or tones which were approximately correct in the prepared sketch.

dark. The last half minute seemed long. My eyes were fixed on the sky line. Suddenly the entire range fell to a deep low-valued blue, and simultaneously the lower part of the sky above the range turned to a rich yellow, inclining to orange, streaked with two horizontal blue-gray clouds. Above me the sky darkened rapidly. For an instant the valley retained its light-green color, and then the shadow seemed to rush toward us and all was engulfed as the call "Go" was shouted. The accompanying color illustration of the approaching Moon shadow ( see Plate XVI) is from a "memory" painting made the next day, the time 10 seconds before totality. 
Turning on my heel, I looked at the corona, blazing steadily in the heavens as if it had always been there. The clear space in the sky had not quite reached the Sun. The thin intervening cloud extended to right and left of the Sun and stood out with its edges illuminated and sharply defined against a velvety night sky of wonderful bluish violet.

Here was a new problem. I had not expected the cloud. I began by drawing the outline of the cloud (slightly nearer the Sun than it actually was so as to get

Figure 3.-Total Solar Ectipse June 8, 1918. Baker, Oregon. Amended Drawing Based on Drawings, Negatives and Positives.

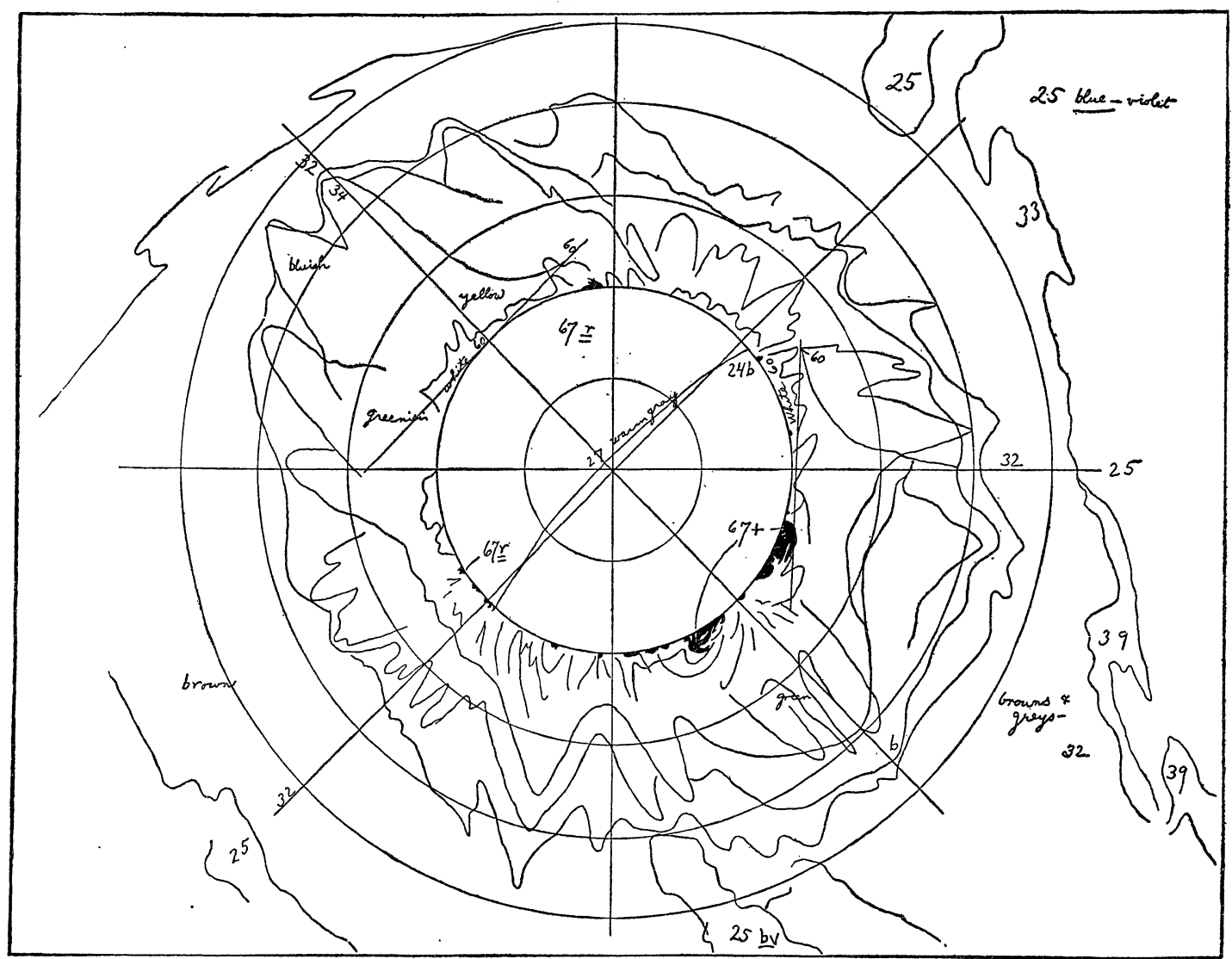

The artist's original drawing as amended later by reference to photographs made of the corona. The details of the polar rays and of the prominences had been left for the cameras to record. Careful drawings of these features and of the variations in shading of the corona resulted in this composite picture on which was based the painting of the corona. The lines outlining the corona in this figure may be regarded as contours of luminosity, showing the range and extent of certain degrees of brilliancy around the disk.

both cloud and sky well on the cardboard), then entered the value and color of the sky as $30 b v$, and the cloud edges which were higher and silvery. The cloud itself, of varying thicknesses, was warmer in tone than the sky and played, I estimated, between 30 and 40 . The Moon was about the same value and much grayer than the sky. I was not conscious of any considerable variations of value in the Moon and failed to put in the value line. The blackishness of the Moon and the center lighter than the edges were undoubtedly optical illusions. Next a quick outline of the corona was made, most attention being paid to the larger rays. Then the binoculars (which had been previously adjusted and focused) were used. Two splendid 
Figure 4.-Total Solar Eclipse June 8, 1918. Baker, Oregon. Values as noted by Howard. Russell Butler.

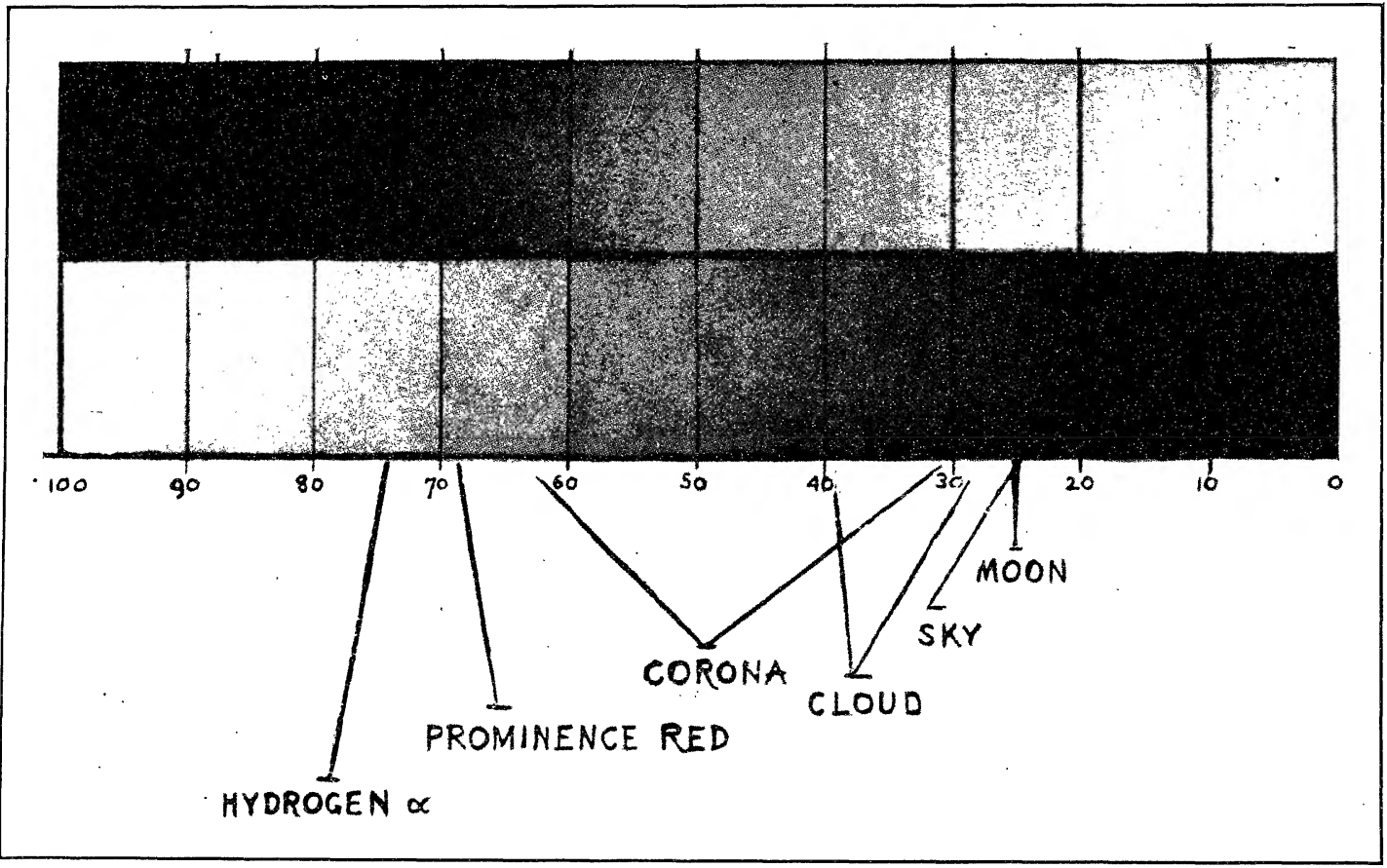

A graphic representation of the scale of brightness values of the various colors found in the eclipse phenomena.-Varnished ivory black is taken as zero and the best white lead (silver white) as 100 for the points of reference. The most brilliant shades were found in the prominences which consist for the most part of incandescent hydrogen gas with a color approaching that of the rod hydrogen line of the spectrum. By careful painting the brightness of the reds used in portraying the prominences was forced up to 67 , and a very fiery quality given to them. The brightness of the sky was pitched at 25 , as was the Moon, while it was estimated that the clouds ranged from 30 to 40 , and the corona from about 30 to 60 . 
prominences, slightly pinker and lighter than I had expected, appeared-one near the top of the Sun and the other on the left side below the horizontal, I gave these the highest value which I then thought could be produced by mixing oil paints, viz, 60r. A rose-colored glow stretched along the lower right side of thelimb, the value of which was first recorded on the chart as 50 .

I recorded two lines of values for the outer corona. I saw no distinct separation of the inner and outer coronas. On the upper left extension greenish and yellowish tones were recorded. No time was wasted on tones thought to be correct in the sample picture. On the whole the corona was less blue than my sample, and it retained brilliancy farther out than I expected. Had it been seen against the blue sky it probably would have extended still farther and its disappearance might have been more gradual. Two sections of the so-called inner corona were very brilliant, although, of course, not as high in value as the prominences. These were next to the limb and were very neutral as to color. I outlined them and marked them "whitish," but got one of them in the wrong place. This brought my eyes to the picture for several seconds. About the ninety-fifth second I looked up and was surprised to see that the pink glow had lengthened out and risen in value. This change was due to the motion of the Moon, which had by that time uncovered a magnificent solar eruption, but I had no time to take up the glasses. I outlined this glow, its value fully up to 60, which I entered afterward. Figure 2 is a reproduction of the original drawing.

Toward the end I reoutlined the corona, indicating rapidly the polar rays, for the accurate drawing of which, as well as for that of the prominences, I intended to rely on the photographs. These rays were decidedly apparent. Suddenly I was blinded by the first of "BAILY's Beads," or the first glimpse of the solar crescent broken by the rough limb of the Moon. It looked like a miniature Sun radiating in all directions. And all was over.

Thanks to the privacy of the grounds and the consideration shown me I was able to proceed at once with my first oil sketch, and for two hours worked uninterruptedly. The next day, June 9, I painted the picture of the approaching Moon shadow over the Elkhorn Range as I remembered it and also a second oil of the corona.

While disappointed in not seeing the corona in a cloudless sky, the thin veil had its advantage from the artist's standpoint. It added mystery and the effect was picturesque. The brilliant corona burned through the thin veil as if it, were not there. Probably only the outside edges of the corona were affected.

On June 10 the photographic negatives were shown to me. Those of the 65-foot camera were $7 \frac{3}{8}$ inches in diameter, the others considerably smaller. I now saw, in minute detail, the two prominences which I had recorded and the mighty cyclone which had been increasingly revealed as the eclipse neared its end, because of the direction of the Moon's motion. We are told that this group of prominences was 46,000 miles high. There were many other minor prominences.

I now made careful drawings of these prominences from the negatives and of the variations in shading of the surrounding corona. Many arches were found springing over the prominences, and a few rifts or dark channels radiating from $101141^{\circ}-26-$ PT II- 64 
the limb but never coming very close to it. The negatives showed very clearly the hairy polar rays, not always radial in direction, and the beginning of a wing springing from the upper right-hand limb of the Sun.

By careful process painting, as already described, I have been able to force up the value of the prominence reds, which appear in Figures 3 and 4 at about 67. I also concluded to reduce the value of the clear sky from 30 to 25 , thus obtaining a range of 42 points instead of 30 , an increase in the ratio of 7 to 5 . In this new scale the other.values take their proportional places. Thus a value of $35(30+5)$ in Figure 1 becomes $32(25+7)$ in Figure 3.

In Figure 3 the corona lines, derived from the drawing and many photographs, may be regarded as a sort of composite, suggesting contours of luminosity very much as contours of elevation appear on a map.

Three paintings were made, the first immediately after the eclipse, the second on the succeeding day, and the third after all data had been secured. This final painting is the one reproduced in conjunction with this article.

Returning with Professor Mrtchell, we stopped at Williams Bay, Wisconsin, and I had the great pleasure and advantage of discussing the problems of the final picture with Prof. E. E. Barnard and Dr. E. B. Frost, of the Yerkes Observatory. They also showed me excellent photographs taken at one of the Yerkes stations and spectroscopic photographs of the prominences taken at the Yerkes Observatory (at the time of totality at the Green River Station), apparently identical as to drawing with those taken at the Baker Station. I wish to acknowledge my indebteriness to these eminent astronomers. 